\title{
Nonlinear Connections and Nearly Autoparallel Maps in General Relativity
}

\author{
Heinz Dehnen* and Sergiu I. Vacaru ${ }^{\dagger}$ \\ Fachbereich Physik, Universitat Konstanz, \\ Postfach M 677, D-78457, Konstanz, Germany
}

August 15, 2000

\begin{abstract}
We apply the method of moving anholonomic frames, with associated nonlinear connections, in (pseudo) Riemannian spaces and examine the conditions when various types of locally anisotropic (la) structures (Lagrange, Finsler like and more general ones) could be modeled in general relativity. New classes of solutions of the Einstein equations with generic local anisotropy are constructed. We formulate the theory of nearly autoparallel (na) maps and introduce the tensorial na-integration as the inverse operation to both covariant derivation and deformation of connections by na-maps. The problem of redefinition of the Einstein gravity theory on na-backgrounds, provided with a set of na-map invariant conditions and local conservation laws, is analyzed. There are illustrated some examples of generation of vacuum Einstein fields by Finsler like metrics and chains of na-maps.
\end{abstract}

\section{Introduction}

It is possible that a corresponding spacetime anholonomic frame structure induces local anisotropies which also 'slightly' modifies the isotropy of cosmic background radiation. Usually, by developing different cosmological scenaria, it is believed that anisotropies and inhomogeneities in the Universe are caused only by anisotropic matter (classical and/or quantum) fluctuations and distributions. To compare some anisotropic effects of two different origins (induced geometrically by imposing some anholonomic conditions or by matter fields anisotropies) it is necessary to perform

\footnotetext{
${ }^{*} \mathrm{e}$-mail: Heinz.Dehnen@uni-konstanz.de

${ }^{\dagger} \mathrm{e}-$ mail: vacaru@lises.asm.md, on leave of absence from the Institute of Applied Physics, Academy of Sciences, Chişinău MD2028, Republic of Moldova
} 
a rigorous definiton and analysis of physical values and fundamental field equations with respect to such anholonomic bases when there are modeled constrained dynamical systems with 'mixed' holonomic and anholonomic variables.

Such models of strings, gravity and matter field locally anisotropic (la) interactions (in brief, we use such terms as la-gravity, la-strings, la-spacetime and so on) have been proposed with the aim to illustrate that the so-called 'locally anisotropic physics' follows alternatively in the low energy energy limits of (super) string theory and that self-consistent theories with both la-spacetime and la-matter could be constructed [36, 37, 38, 39]. There are developed different approaches to laspacetimes and la-gravity which are grounded on Finsler geometry and generalizations [14, 10, 31, 25, 1, 2, 27, 28, 44. For instance, there is a subclass of models with local anisotropy which try to describe possible violations of local Lorentz invariance in the framework of Finsler geometry [5, 6, 16]. This non infrequently introduces the misunderstanding that an 'unusual' relativity is presented in all Finsler like theories and lied to the misinterpretation that experimentally such Finsler spaces met rather stringent constrains 45].

A surprising result is that Finsler like metrics and their generalizations could be found as solutions of the Einstein equations in general relativity and higher dimension gravity models (see 41] and Sections 2 and 8 in this work). The point is to model various type of la-structures (Finsler type and more general ones) by using anholonomic frames on (pseudo) Riemannian spacetimes. This class of la-spacetimes are compatible with the paradigm of the Einstein-Lorenz-Poincare relativity and the theory of experiment on such curved spacetimes have to be adapted to the viewpoint of observers stated with respect to anholonomic frames of reference.

The problem of equivalence of spaces with generalized metrics and connections was considered in a series of works by E. Cartan [7, 8, 9, 10, 11, 12] who developed an unified approach to Riemannian, affine and projective connection, fiber bundles, Finsler and another type of curved spaces on the language of moving frames and differential forms. Paper [10] contains also the idea on nonlinear connection (N-connection) associated to an anholonomic frame. The global definition of $\mathrm{N}-$ connection is due to W. Barthel [3] and this concept was developed and applied by R. Miron and M. Anastasiei 27, 28] in their geometry of generalized Lagrange and Finsler spaces modeled on vector and tangent bundles. The further, in this line, geometric extensions and applications in physics are connected with the (super) frames, metrics and connections in spinor spaces and superbundles provided with $\mathrm{N}$-connection structures and the geometry of locally anisotropic strings and gravity [36, 39, 37, 38.

The first purpose of this paper is to demonstrate that anholonomic frame structures with associated $\mathrm{N}$-connections on (pseudo) Riemannian spacetimes display a new 'locally anisotropic' picture of the Einstein gravity. Here is to be noted that the elaboration of models with la-interactions is considered to entail great difficulties because of the problematical character of the possibility and manner of definition of conservation laws on la-spaces. It will be recalled that, for instance, in special relativity the conservation laws of energy-momentum type are defined by the global group of automorphisms (the Poincare group) of the fundamental Mikowski spaces. 
For (pseudo) Riemannian spaces one has only tangent space's automorphisms and for particular cases there are symmetries generated by Killing vectors. No global or local automorphisms exist on generic la-spaces and in result of this fact the formulation of la-conservation laws is sophisticate and full of ambiguities. Nevertheless, a variant of definition of energy-momentum values for gravitational and matter lafields and the form of conservation laws for such values is possible if we introduce moving frames and consider that anisotropies are effectively modeled on (pseudo) Riemannian spacetimes.

The second aim of this paper is to develop a necessary geometric background (the theory of nearly autoparallel maps, in brief, na-maps, and tensor integral formalism on multispaces) for formulation and a detailed investigation of conservation laws on locally isotropic and/or anisotropic curved spaces. We shall adapt for the la-spacetimes induced by anholonomic structures some results from the theory of na-maps for generalized affine spaces, Einstein-Cartan and Einstein spaces, fibre bundles and different subclasses of generic la-spaces (see [43, 18, 17] and 40] as reviews of our results published in some less accessible books and journals from former URSS and Romania).

The question of definition of tensor integration as the inverse operation of covariant derivation was posed and studied by A.Moór [30]. Tensor-integral and bitensor formalisms turned out to be very useful in solving certain problems connected with conservation laws in general relativity [17, 40]. In order to extend tensor-integral constructions we proposed to take into consideration nearly autoparallel and nearly geodesic [32, 40] maps (in brief, we write ng-maps, ng-theory) which forms a subclass of local 1-1 maps of curved spaces with deformation of the connection and metric structures. The third purpose of this work is to synthesize the results on namaps and multispace tensor integrals, to reformulate them for anholonomic (pseudo) Riemannian spacetimes and to propose a variant of definition of conservation laws and energy-momentum type values on la-spacetimes.

Our investigations are completed by some explicit examples of new solutions of Einstein equations in general relativity which admit nearly autoparallel maps and/or Finsler like structures.

The paper is organized as follows: Section 2 outlines the geometry of anholonomic frames with associated nonlinear connection structures. The general criteria when a Finsler like metric could be embedded into the Einstein gravity is formulated. Section 3 is devoted to the theory of nearly autoparallel (na) maps of la-spacetimes. The classification of na-maps and corresponding invariant conditions are given in Section 4. In Section 5 we define the nearly autoparallel tensor-integral on locally anisotropic multispaces. The problem of formulation of conservation laws on spaces with local anisotropy is studied in Section 6. We present a definition of conservation laws for la-gravitational fields on na-images of la-spaces in Section 7. Some new classes of vacuum and non-vacuume solutions of the Einstein equations, induced by Finsler like metrics, are constructed in Section 8. In Section 9 we illustrate how a class of vacuum Einstein fields with Finsler like structures can be mapped via chains of na-transforms to the flat Minkowski spacetime. The results are outlined in Section 10. 


\section{Anholonomic Frames and Anisotropic Metrics}

We outline the geometric background of the Einstein gravity with respect to anholonomic frames and associated nonlinear connections ( $\mathrm{N}$-connection) modelling $m$ dimensional local anisotropies in (pseido) Riemannian $(n+m)$-dimensional spacetimes. Comparing with another approaches concerning anholonomic frames (tetrads or vierbiends, in four dimensions) in general relativity (see, for instance, [13, 29, 26]), it should be noted that topic of application of $\mathrm{N}$-connections in general relativity and Kaluza-Klein theories was not considered in the well known monographs and works. The $\mathrm{N}$-connection geometry was investigated in details, from the viewpoint of modelling of generalized Lagrange and Finsler geometries in vector and tangent bundle spaces, in the monographs [27, 28, 14] and, with applications to locally anisotropic (la) spinor differential geometry, supergravity and superstrings in [36, 39, 37, 38]. We pointed to the positive necessity to consider anholonomic frames with associated $\mathrm{N}-$ connections (with mixed holonomic and anholonomic degrees of friedom) in order to develop self-consistent relativistic theories with generic anisotropy, anisotropic distributions of matter and field interactions, kinetic and thermodynamic processes on (pseudo) Riemannian spacetimes in [41]. The final purpose of this Section is to proof that la-structures (Finsler like or more general ones) could be induced in general relativity.

\subsection{Anholonomy, local anisotropy, and Einstein equations}

In this work spacetimes are modeled as smooth (i. e. class $\left.C^{\infty}\right)(n+m)$-dimensional (pseudo) Riemannian manifolds $V^{(n+m)}$ being Hausdorff, paracompact and connected. A spacetime $V^{(n+m)}$ is enabled with the fundamental structures of symmetric metric $g_{\alpha \beta}$ and of linear, in general nonsymmetric (if we consider anholonomic frames), connection $\Gamma_{\beta \gamma}^{\alpha}$ defining the covariant derivation $D_{\alpha}$ which is chosen to satisfy the metricity conditions $D_{\alpha} g_{\beta \gamma}=0$. The indices of geometrical objects are given with respect to a frame vector field $\delta^{\alpha}=\left(\delta^{i}, \delta^{a}\right)$ and its dual $\delta_{\alpha}=\left(\delta_{i}, \delta_{a}\right)$. For instance, a covariant-contravariant tensor $Q$ is decomposed as

$$
Q=Q_{\alpha}^{\beta} \delta^{\alpha} \otimes \delta_{\beta}
$$

where $\otimes$ is the tensor product. A holonomic frame structure on $V^{(n+m)}$ could be stated by a local coordinate base

$$
\partial_{\alpha}=\partial / \partial u^{\alpha}
$$

consisting from usual partial derivative components, and the dual basis

$$
d^{\alpha}=d u^{\alpha}
$$

consisting from usual differentials. An arbitrary holonomic frame $e_{\alpha}$ could be related

to a coordinate one by a local linear transform $e_{\alpha}=A_{\alpha}{ }^{\beta} \partial_{\beta}$, for which the matrix $A_{\alpha}{ }^{\beta}$ is nondegenerate and there are satisfied the holonomy conditions, $e_{\alpha} e_{\beta}-e_{\beta} e_{\alpha}=0$. 
Let us consider a $(n+m)$-dimensional metric parametrized

$$
g_{\alpha \beta}=\left[\begin{array}{cc}
g_{i j}+N_{i}^{a} N_{j}^{b} h_{a b} & N_{j}^{e} h_{a e} \\
N_{i}^{e} h_{b e} & h_{a b}
\end{array}\right]
$$

with respect to a local coordinate basis (2), $d u^{\alpha}=\left(d x^{i}, d y^{a}\right)$, where the Greek indices run values $1,2, \ldots, n+m$, the Latin indices $i, j, k, \ldots$ from the middle of the alphabet run values $1,2, \ldots, n$ and the Latin indices from the beginning of the alphabet, $a, b, c, \ldots$, run values $1,2, \ldots, m$. The coefficients $g_{i j}=g_{i j}\left(u^{\alpha}\right), h_{a e}=h_{a e}\left(u^{\alpha}\right)$ and $N_{i}^{a}=N_{i}^{a}\left(u^{\alpha}\right)$ will be defined by a solution of the Einstein gravitational field equations.

The metric (3) can be rewritten in a block $(n \times n) \oplus(m \times m)$ form

$$
g_{\alpha \beta}=\left(\begin{array}{cc}
g_{i j}\left(u^{\alpha}\right) & 0 \\
0 & h_{a b}\left(u^{\alpha}\right)
\end{array}\right)
$$

with respect to the anholonomic bases

$$
\delta_{\alpha}=\left(\delta_{i}, \partial_{a}\right)=\frac{\delta}{\partial u^{\alpha}}=\left(\delta_{i}=\frac{\delta}{\partial x^{i}}=\frac{\partial}{\partial x^{i}}-N_{i}^{b}\left(u^{\alpha}\right) \frac{\partial}{\partial y^{b}}, \partial_{a}=\frac{\partial}{\partial y^{a}}\right)
$$

and

$$
\delta^{\beta}=\left(d^{i}, \delta^{a}\right)=\delta u^{\beta}=\left(d^{i}=d x^{i}, \delta^{a}=\delta y^{a}=d y^{a}+N_{k}^{a}\left(u^{\alpha}\right) d x^{k}\right) .
$$

where the coefficients $N_{j}^{a}\left(u^{\alpha}\right)$ from (5) and (6) are treated as the components of an associated nonlinear connection, $\mathrm{N}$-connection, structure [3, 27, 28, 36, 39, 38].

A frame (local basis) structure $\delta_{\alpha}$ on $V^{(n+m)}$ is characterized by its anholonomy coefficients $w_{\beta \gamma}^{\alpha}$ defined from relations

$$
\delta_{\alpha} \delta_{\beta}-\delta_{\beta} \delta_{\alpha}=w_{\alpha \beta}^{\gamma} \delta_{\gamma}
$$

The rigorous mathematical definition of $\mathrm{N}$-connection is based on the formalism of horizontal and vertical subbundles and on exact sequences in vector bundles [3], 28]. In this work we introduce a $\mathrm{N}$-connection as a distribution which for every point $u=(x, y) \in V^{(n+m)}$ defines a local decomposition of the tangent space

$$
T_{u} V^{(n+m)}=H_{u} V^{(n)} \oplus V_{u} V^{(m)} .
$$

into horizontal, $H_{u} V^{(n)}$, and vertical (anisotropy), $V_{u} V^{(m)}$, subspaces which is given by a set of coefficients $N_{j}^{a}\left(u^{\alpha}\right)$.

A $\mathrm{N}$-connection is characterized by its curvature

$$
\Omega_{i j}^{a}=\frac{\partial N_{i}^{a}}{\partial x^{j}}-\frac{\partial N_{j}^{a}}{\partial x^{i}}+N_{i}^{b} \frac{\partial N_{j}^{a}}{\partial y^{b}}-N_{j}^{b} \frac{\partial N_{i}^{a}}{\partial y^{b}} .
$$

Here we note that the class of usual linear connections can be considered as a particular case of $\mathrm{N}$-connections when

$$
N_{j}^{a}(x, y)=\Gamma_{b j}^{a}(x) y^{b}
$$


The elongation (by $\mathrm{N}$-connection) of partial derivatives and differentials in the adapted to the $\mathrm{N}$-connection operators (5) and (6) reflects the fact that on the (pseudo) Riemannian spacetime $V^{(n+m)}$ it is modeled a generic local anisotropy characterized by anholonomy relations (17) when the anolonomy coefficients are computed as follows

$$
\begin{aligned}
& w^{k}{ }_{i j}=0, w^{k}{ }_{a j}=0, w^{k}{ }_{i a}=0, w^{k}{ }_{a b}=0, w^{c}{ }_{a b}=0, \\
& w_{i j}^{a}=-\Omega_{i j}^{a}, w^{b}{ }_{a j}=-\partial_{a} N_{i}^{b}, w^{b}{ }_{i a}=\partial_{a} N_{i}^{b} .
\end{aligned}
$$

The frames (5) and (6) are locally adapted to the $\mathrm{N}$-connection structure, define a local anisotropy and, in brief, are called la-bases.

A N-connection structure distinguishes the geometrical objects into horizontal and vertical components. Such objects are briefly called d-tensors, d-metrics and $\mathrm{d}$-connections. Their components are defined with respect to a la-basis of type (5), its dual (6), or their tensor products (d-linear or d-affine transforms of such frames could also be considered). For instance, a covariant and contravariant d-tensor $Q$, is expressed

$$
Q=Q^{\alpha}{ }_{\beta} \delta_{\alpha} \otimes \delta^{\beta}=Q^{i}{ }_{j} \delta_{i} \otimes d^{j}+Q^{i}{ }_{a} \delta_{i} \otimes \delta^{a}+Q^{b}{ }_{j} \partial_{b} \otimes d^{j}+Q^{b}{ }_{a} \partial_{b} \otimes \delta^{a} .
$$

In this paper, as a locally anisotropic spacetime, la-spacetime, we shall consider a pseudo-Riemannian spacetime provided with a metric of signature $(-,+$, $+,+)$ (a permutation of signes being also possible) and with an anholonomic frame basis defined by an associated $\mathrm{N}$-connection structure when the coefficients of the mentioned objects are imposed to be a solution of the Einstein equations.

A linear d-connection $D$ on la-spacetime $V^{(n+m)}$,

$$
D_{\delta_{\gamma}} \delta_{\beta}=\Gamma_{\beta \gamma}^{\alpha}(x, y) \delta_{\alpha},
$$

is given by its $\mathrm{h}-\mathrm{v}-$ components,

$$
\Gamma_{\beta \gamma}^{\alpha}=\left(L_{j k}^{i}, L_{b k}^{a}, C_{j c}^{i}, C_{b c}^{a}\right)
$$

where

$$
D_{\delta_{k}} \delta_{j}=L^{i}{ }_{j k} \delta_{i}, D_{\delta_{k}} \partial_{b}=L_{b k}^{a} \partial_{a}, D_{\partial_{c}} \delta_{j}=C^{i}{ }_{j c} \delta_{i}, D_{\delta_{c}} \partial_{b}=C_{b c}^{a} \partial_{a} .
$$

A metric on $V^{(n+m)}$ with its coefficients parametrized as (3) can be written in distingushed form (4), as a metric d-tensor (in brief, d-metric), with respect to a la-base (6), i. e.

$$
\delta s^{2}=g_{\alpha \beta}(u) \delta^{\alpha} \otimes \delta^{\beta}=g_{i j}(x, y) d x^{i} d x^{j}+h_{a b}(x, y) \delta y^{a} \delta y^{b} .
$$

Some $\mathrm{N}$-connection, $\mathrm{d}-$-connection and $\mathrm{d}$-metric structures are compatible if there are satisfied the conditions

$$
D_{\alpha} g_{\beta \gamma}=0 \text {. }
$$

For instance, a canonical compatible d-connection

$$
{ }^{c} \Gamma_{\beta \gamma}^{\alpha}=\left({ }^{c} L^{i}{ }_{j k},{ }^{c} L_{b k}^{a},{ }^{c} C^{i}{ }_{j c},{ }^{c} C^{a}{ }_{b c}\right)
$$


is defined by the coefficients of $\mathrm{d}$-metric (10), $g_{i j}(x, y)$ and $h_{a b}(x, y)$, and by the coefficients of $\mathrm{N}$-connection,

$$
\begin{aligned}
{ }^{c} L^{i}{ }_{j k} & =\frac{1}{2} g^{i n}\left(\delta_{k} g_{n j}+\delta_{j} g_{n k}-\delta_{n} g_{j k}\right) \\
{ }^{c} L^{a}{ }_{b k} & =\partial_{b} N_{k}^{a}+\frac{1}{2} h^{a c}\left(\delta_{k} h_{b c}-h_{d c} \partial_{b} N_{i}^{d}-h_{d b} \partial_{c} N_{i}^{d}\right) \\
{ }^{c} C^{i}{ }_{j c} & =\frac{1}{2} g^{i k} \partial_{c} g_{j k}, \\
{ }^{c} C_{b c}^{a} & =\frac{1}{2} h^{a d}\left(\partial_{c} h_{d b}+\partial_{b} h_{d c}-\partial_{d} h_{b c}\right)
\end{aligned}
$$

The coefficients of the canonical d-connection generalize for la-spacetimes the well known Cristoffel symbols. By a local linear nondegenerate transform to a coordinate frame we obtain the coefficients of the usual (pseudo) Riemannian metric connection.

For a canonical d-connection (9) the components of canonical torsion,

$$
\begin{aligned}
T\left(\delta_{\gamma}, \delta_{\beta}\right) & =T_{\beta \gamma}^{\alpha} \delta_{\alpha}, \\
T_{\beta \gamma}^{\alpha} & =\Gamma_{\beta \gamma}^{\alpha}-\Gamma_{\gamma \beta}^{\alpha}+w_{\beta \gamma}^{\alpha}
\end{aligned}
$$

are expressed via $\mathrm{d}$-torsions

$$
\begin{aligned}
& T_{. j k}^{i}=T_{j k}^{i}=L_{j k}^{i}-L_{k j}^{i}, \quad T_{j a}^{i}=C_{. j a}^{i}, T_{a j}^{i}=-C_{j a}^{i} \\
& T_{. j a}^{i}=0, \quad T_{. b c}^{a}=S_{. b c}^{a}=C_{b c}^{a}-C_{c b}^{a}, \\
& T_{. i j}^{a}=-\Omega_{i j}^{a}, \quad T_{. b i}^{a}=\partial_{b} N_{i}^{a}-L_{. b j}^{a}, \quad T_{. i b}^{a}=-T_{. b i}^{a}
\end{aligned}
$$

which reflects the anholonomy of the corresponding la-frame of reference on $V^{(n+m)}$; they are induced effectively. With respect to holonomic frames the d-torsions vanishes.

For simplicity, hereafter, we shall omit the up left index " $c$ " and consider only connections and $\mathrm{d}$-connections defined by compatible metric and $\mathrm{N}$-connection coefficients.

Putting the non-vanishing coefficients (9) into the formula for curvature

$$
\begin{aligned}
R\left(\delta_{\tau}, \delta_{\gamma}\right) \delta_{\beta} & =R_{\beta \gamma \tau}^{\alpha} \delta_{\alpha} \\
R_{\beta \gamma \tau}^{\alpha} & =\delta_{\tau} \Gamma_{\beta \gamma}^{\alpha}-\delta_{\gamma} \Gamma_{\beta \delta}^{\alpha}+\Gamma_{\beta \gamma}^{\varphi} \Gamma_{\varphi \tau}^{\alpha}-\Gamma_{\beta \tau}^{\varphi} \Gamma_{\varphi \gamma}^{\alpha}+\Gamma_{\beta \varphi}^{\alpha} w_{\gamma \tau}^{\varphi}
\end{aligned}
$$

we compute the components of canonical d-curvatures

$$
\begin{aligned}
R_{h . j k}^{i} & =\delta_{k} L_{. h j}^{i}-\delta_{j} L_{. h k}^{i}+L_{. h j}^{m} L_{m k}^{i}-L_{. h k}^{m} L_{m j}^{i}-C_{. h a}^{i} \Omega_{. j k}^{a}, \\
R_{b . j k}^{a} & =\delta_{k} L_{. b j}^{a}-\delta_{j} L_{. b k}^{a}+L_{. b j}^{c} L_{. c k}^{a}-L_{. b k}^{c} L_{. c j}^{a}-C_{. b c}^{a} \Omega_{. j k}^{c}, \\
P_{j . k a}^{. i} & =\partial_{k} L_{. j k}^{i}+C_{. j b}^{i} T_{. k a}^{b}-\left(\partial_{k} C_{. j a}^{i}+L_{. l k}^{i} C_{. j a}^{l}-L_{. j k}^{l} C_{. l a}^{i}-L_{. a k}^{c} C_{. j c}^{i}\right), \\
P_{b . k a}^{. c} & =\partial_{a} L_{. b k}^{c}+C_{. b d}^{c} T_{. k a}^{d}-\left(\partial_{k} C_{. b a}^{c}+L_{. d k}^{c} C_{. b a}^{d}-L_{. b k}^{d} C_{. d a}^{c}-L_{. a k}^{d} C_{. b d}^{c}\right), \\
S_{j . b c}^{. i} & =\partial_{c} C_{. j b}^{i}-\partial_{b} C_{. j c}^{i}+C_{. j b}^{h} C_{. h c}^{i}-C_{. j c}^{h} C_{h b}^{i}, \\
S_{b . c d}^{a} & =\partial_{d} C_{. b c}^{a}-\partial_{c} C_{. b d}^{a}+C_{. b c}^{e} C_{. e d}^{a}-C_{. b d}^{e} C_{. e c}^{a} .
\end{aligned}
$$


The Ricci d-tensor $R_{\beta \gamma}=R_{\beta \gamma \alpha}^{\alpha}$ has the components

$$
\begin{aligned}
& R_{i j}=R_{i . j k}^{. k}, \quad R_{i a}=-{ }^{2} P_{i a}=-P_{i . k a}^{. k} \\
& R_{a i}={ }^{1} P_{a i}=P_{a . i b}^{. b}, \quad R_{a b}=S_{a . b c}^{. c}
\end{aligned}
$$

and, in general, this d-tensor is non symmetric.

We can compute the scalar curvature $\overleftarrow{R}=g^{\beta \gamma} R_{\beta \gamma}$ of a d-connection $D$

$$
\overleftarrow{R}=\widehat{R}+S
$$

where $\widehat{R}=g^{i j} R_{i j}$ and $S=h^{a b} S_{a b}$.

By introducing the values (13) and (14) into the usual Einstein equations

$$
R_{\beta \gamma}-\frac{1}{2} g_{\beta \gamma} R=k \Upsilon_{\beta \gamma},
$$

written with respect to an anholonomic la-frame of reference, we obtain the system of field equations for la-gravity with $\mathrm{N}$-connection structure 28]:

$$
\begin{aligned}
R_{i j}-\frac{1}{2}(\widehat{R}+S) g_{i j} & =k \Upsilon_{i j}, \\
S_{a b}-\frac{1}{2}(\widehat{R}+S) h_{a b} & =k \Upsilon_{a b}, \\
{ }^{1} P_{a i} & =k \Upsilon_{a i}, \\
{ }^{2} P_{i a} & =-k \Upsilon_{i a},
\end{aligned}
$$

where $\Upsilon_{i j}, \Upsilon_{a b}, \Upsilon_{a i}$ and $\Upsilon_{i a}$ are the components of the energy-momentum d-tensor field $\Upsilon_{\beta \gamma}$ which includes the cosmological constant terms and possible contributions of $\mathrm{d}$-torsions and matter, and $k$ is the coupling constant.

\subsection{Finsler like metrics in Einstein gravity}

In this subsection we follow the almost Hermitian model of Finsler geometry [27, 28] and consider a $V^{2 n}$ (pseudo) Riemannian spacetime.

The locally anisotropic structure is modeled on the manifold $\widetilde{T V}=T V^{(n)} \backslash\{0\}$, where $\backslash\{0\}$ means that there is eliminated the null cross-section of the bundle projection $\left.\tau: T V^{(n)} \rightarrow V^{(n)}\right)$. There are considered d-metrics of type (10) with identical $(n \times n)$-dimensional blocks for both base and fiber components. On $T V^{(n)}$ we can define a natural almost complex structure $C_{(a)}$,

$$
C_{(a)}\left(\delta_{i}\right)=-\partial / \partial y^{i} \text { and } C_{(a)}\left(\partial / \partial y^{i}\right)=\delta_{i}
$$

where the la-derivatives (5) and la-differentials act on the bundle $\widetilde{T V}$ being adapted to a nontrivial $\mathrm{N}$-connection $N=\left\{N_{j}^{k}(x, y)\right\}$ in $T V$ and $C_{(a)}^{2}=-I$. The pair $\left(\delta s^{2}, C_{(a)}\right)$ defines an almost Hermitian structure on $\widetilde{T V}$ with an associate 2 -form

$$
\theta=h_{i j}(x, y) \delta^{i} \wedge d x^{j}
$$


and the triad $K^{2 n}=\left(\widetilde{T V}, \delta s^{2}, C_{(a)}\right)$ is an almost Kählerian space. We can verify that the canonical $\mathrm{d}$-connection (9) satisfies the conditions

$$
{ }^{c} D_{X}\left(\delta s^{2}\right)=0,{ }^{c} D_{X}\left(C_{(a)}\right)=0
$$

for any d-vector $X$ on $T V^{(n)}$ and has zero $h h h$ - and $v v v$-torsions (where $\mathrm{h}$ - and $\mathrm{v}-$ denote the horizontal and vertical components).

The notion of Lagrange space [22, 27, 28] was introduced as a generalization of Finsler geometry in order to geometrize the fundamental concepts in mechanics. A regular Lagrangian $L\left(x^{i}, y^{i}\right)$ on $\widetilde{T V}$ is defined by a continuity class $C^{\infty}$ function $L: T V^{(n)} \rightarrow \mathbb{R}$ for which the matrix

$$
h_{i j}(x, y)=\frac{1}{2} \frac{\partial^{2} L}{\partial y^{i} \partial y^{j}}
$$

has the rank $n$. A d-metric (10) with coefficients of form (16), a corresponding canonical d-connection (9) and almost complex structure $C_{(a)}$ defines an almost Hermitian model of Lagrange geometry.

Metrics $h_{i j}(x, y)$ of rank $n$ and constant signature on $\widetilde{T V}$, which can not be determined as a second derivative of a Lagrangian are considered in the so-called generalized Lagrange geometry on $T V^{(n)}$ (see details in [27, 28]).

A subclass of metrics of type (16) consists from those where instead of a regular Lagrangian one considers a Finsler metric function $F$ on $V^{(n)}$ defined as $F: T V^{(n)} \rightarrow$ $\mathbb{R}$ having the properties that it is of class $C^{\infty}$ on $\widetilde{T V}$ and only continuous on the image of the null cross-section in $T V^{(n)}$, the restriction of $F$ on $\widetilde{T V}$ is a positive function homogeneous of degree 1 with respect to the variables $y^{i}$, i. e.

$$
F(x, \lambda y)=\lambda F(x, y), \lambda \in \mathbb{R},
$$

and the quadratic form on $\mathbb{R}^{2}$, with coefficients

$$
h_{i j}(x, y)=\frac{1}{2} \partial^{2} F^{2} / \partial y^{i} \partial y^{j}
$$

(see $(10)$ ) given on $\widetilde{T M}$, is positive definite.

Very different approaches to Finsler geometry, its generalizations and applications are examined in a number of monographs [14, 10, 31, 1, 2, 4, 6, 27] and it is considered that for such geometries the usual (pseudor)Riemannian metric interval

$$
d s=\sqrt{g_{i j}(x) d x^{i} d x^{j}}
$$

on a manifold $M$ is changed into a nonlinear one

$$
d s=F\left(x^{i}, d x^{j}\right)
$$

defined by the Finsler metric $F$ (fundamental function) on $\widetilde{T M}$ (it should be noted an ambiguity in terminology used in monographs on Finsler geometry and on gravity theories with respect to such terms as Minkowski space, metric function and so on). 
Geometric spaces with a 'combersome' variational calculus and a number of curvatures, torsions and invariants connected with nonlinear metric intervals of type (16) are considered as less suitable for purposes of the modern field and particle physics.

In our approach to generalized Finsler geometries in (super) string, gravity and gauge theories [38, 40] we advocated the idea that instead of usual geometric constructions based on straightforward applications of derivatives of (17) following from a nonlinear interval (18) one should consider d-metrics (10) with coefficients of necessity determined via an almost Hermitian model of a Lagrange (16), Finsler geometry (17) and/or their extended variants. This way, by a synthesis of the moving frame method with the geometry of $\mathrm{N}$-connections, we can investigate in a unified manner a various class of higher and lower dimension gravitational models with generic, or induced, anisotropies on some anholonomic and/or Kaluza-Klein spacetimes.

Now we analyze the possibility to include $n$-dimensional Finsler metrics into $2 n-$ dimensional (pseudo) Riemannian spaces and formulate the general criteria when a Finsler like metric could be imbedded into the Einstein theory.

Let consider on $\widetilde{T V}$ an ansatz of type (3) when

$$
g_{i j}=\frac{1}{2} \partial^{2} F_{*}^{2} / \partial y^{i} \partial y^{j} \text { and } h_{i j}=\frac{1}{2} \partial^{2} F^{2} / \partial y^{i} \partial y^{j}
$$

i.e.

$$
g_{\widehat{\alpha} \widehat{\beta}}=\frac{1}{2}\left(\begin{array}{cc}
\frac{\partial^{2} F_{*}^{2}}{\partial y^{i} \partial y^{j}}+N_{i}^{k} N_{j}^{l} \frac{\partial^{2} F^{2}}{\partial y^{k} \partial y^{l}} & N_{j}^{l} \frac{\partial^{2} F^{2}}{\partial y^{k} \partial y^{l}} \\
N_{i}^{k} \frac{\partial^{2} F^{2}}{\partial y^{k} \partial y^{l}} & \frac{\partial^{2} F^{2}}{\partial y^{i} \partial y^{j}}
\end{array}\right) .
$$

A metric $g_{\widehat{\alpha} \widehat{\beta}}$ of signature $(-,+, \ldots,+)$ induced by two Finsler functions $F_{*}$ and $F$ (17) (as a particular case $F_{*}=F$ ) is to be treated in the framework of general relativity theory if this metric is a solution of the Einstein equations on a $2 n-$ dimensional (pseudo) Riemannian spacetime written with respect to a holonomic frame. Here we note that, in general, a $\mathrm{N}$-connection on a Finsler space, subjected to the condition that the induced (pseudo) Riemannian metric is a solution of usual Einstein equations, does not coincide with the well known Cartan's N-connection in Finsler geometry [10, 31]. In such cases we have to examine possible compatible deformations of $\mathrm{N}$-connection structures [27, 28].

We can also introduces ansatzs of type (3) with $g_{i j}$ and $h_{i j}$ induced by a Lagrange quadratic form (16). In Section 8 we shall construct solutions of the Einstein equations forllowing from an ansatz for a generalized Finsler metric,

$$
g_{\widehat{\alpha} \widehat{\beta}}=\frac{1}{2}\left(\begin{array}{cc}
\frac{\partial^{2} F_{*}^{2}}{\partial y^{i} \partial y^{j}}+N_{i}^{k} N_{j}^{l} \Lambda_{i j} & N_{j}^{l} \Lambda_{k l} \\
N_{i}^{k} \Lambda_{k l} & \Lambda_{i j}
\end{array}\right),
$$

where the $2 \times 2$ matrix $\Lambda$ is induced by a Finsler metric via a transform

$$
\Lambda_{k l}=\left(C^{T}\right)_{k i} \frac{\partial^{2} F^{2}}{\partial y^{i} \partial y^{j}}(C)_{j l},
$$

parametrized by a $2 \times 2$ matrix $(C)\left(x^{i}, y^{k}\right)$ and its transposition $\left(C^{T}\right)\left(x^{i}, y^{k}\right)$. A general approach to the geometry of spacetimes with generic local anisotropy can be 
developed on imbeddings into corresponding Kaluza-Klein theories and adequate modelling of la-interactions with respect to anholonomic or holonomic frames and associated $\mathrm{N}$-connection structures. As a matter of principle every type of Finsler, Lagrange or generalized Lagrange geometry could be modeled on a corresponding Kaluza-Klein spacetime.

\section{$3 \quad$ Nearly Autoparallel Maps}

The aim of this Section is to formulate the theory of nearly geodesic maps (ng-maps) [32] and nearly autoparallel maps (na-maps) [40, 43] for (pseudo) Riemannian spacetimes provided with anhonlonomic frame and associated $\mathrm{N}$-connection structures.

Our geometric arena consists from pairs of open regions $(U, \underline{U})$ of two la-spacetimes, $U \subset V^{(n+m)}$ and $\underline{U} \subset \underline{V}^{(n+m)}$, and 1-1 local maps $f: U \rightarrow \underline{U}$ given by some functions $f^{\alpha}(u)$ of smoothly class $C^{r}(U),(r>2$, or $r=\omega$ for analytic functions $)$ and their inverse functions $f^{\underline{\alpha}}(\underline{u})$ with corresponding non-zero Jacobians in every point $u \in U$ and $\underline{u} \in \underline{U}$.

We consider that two open regions $U$ and $\underline{U}$ are attributed to a common for $\mathrm{f}$-map coordinate system if this map is realized on the principle of coordinate equality $q\left(u^{\alpha}\right) \rightarrow \underline{q}\left(u^{\alpha}\right)$ for every point $q \in U$ and its f-image $\underline{q} \in \underline{U}$. We note that all calculations included in this work will be local in nature and taken to refer to open subsets of mappings of type $V^{(n+m)} \supset U \stackrel{f}{\longrightarrow} \underline{U} \subset \underline{V}^{(n+m)}$. For simplicity, we suppose that in a fixed common coordinate system for $U$ and $\underline{U}$ the spacetimes $V^{(n+m)}$ and $\underline{V}^{(n+m)}$ are characterized by a common $\mathrm{N}$-connection structure, when

$$
N_{j}^{a}(u)=\underline{N}_{j}^{a}(u)=\underline{N}_{j}^{a}(\underline{u}),
$$

which leads to the possibility to establish common local bases, adapted to a given $\mathrm{N}$-connection, on both regions $U$ and $\underline{U}$. We consider that on $V^{(n+m)}$ it is defined a linear d-connection structure with components $\Gamma_{. \beta \gamma}^{\alpha}$. On the space $\underline{V}^{(n+m)}$ the linear $\mathrm{d}$-connection is considered to be a general one with torsion

$$
\underline{T}_{. \beta \gamma}^{\alpha}=\underline{\Gamma}_{. \beta \gamma}^{\alpha}-\underline{\Gamma}_{. \gamma \beta}^{\alpha}+w_{. \beta \gamma}^{\alpha}
$$

and nonmetricity

$$
\underline{K}_{\alpha \beta \gamma}=\underline{D}_{\alpha} \underline{g}_{\beta \gamma} .
$$

As a particular case we can consider maps to (pseudo) Riemannian spacetimes, when $\underline{K}_{\alpha \beta \gamma}=0$.

Geometrical objects on $\underline{V}^{(n+m)}$ are specified by underlined symbols (for example, $\underline{A}^{\alpha}, \underline{B}^{\alpha \beta}$ ) or by underlined indices (for example, $A^{\underline{a}}, B^{\underline{a b}}$ ).

For our purposes it is convenient to introduce auxiliary symmetric d-connections, $\gamma_{. \beta \gamma}^{\alpha}=\gamma_{. \gamma \beta}^{\alpha}$ on $V^{(n+m)}$ and $\underline{\gamma}_{. \beta \gamma}^{\alpha}=\underline{\gamma}_{. \gamma \beta}^{\alpha}$ on $\underline{V}^{(n+m)}$ defined, correspondingly, as

$$
\Gamma_{. \beta \gamma}^{\alpha}=\gamma_{. \beta \gamma}^{\alpha}+T_{. \beta \gamma}^{\alpha} \text { and } \underline{\Gamma}_{. \beta \gamma}^{\alpha}=\underline{\gamma}_{. \beta \gamma}^{\alpha}+\underline{T}_{. \beta \gamma}^{\alpha} \text {. }
$$


We are interested in definition of local 1-1 maps from $U$ to $\underline{U}$ characterized by symmetric, $P_{. \beta \gamma}^{\alpha}$, and antisymmetric, $Q_{. \beta \gamma}^{\alpha}$, deformations:

$$
\underline{\gamma}_{. \beta \gamma}^{\alpha}=\gamma_{. \beta \gamma}^{\alpha}+P_{. \beta \gamma}^{\alpha}
$$

and

$$
\underline{T}_{. \beta \gamma}^{\alpha}=T_{. \beta \gamma}^{\alpha}+Q_{. \beta \gamma}^{\alpha} .
$$

The auxiliary linear covariant derivations induced by $\gamma_{\beta \gamma}^{\alpha}$ and $\underline{\gamma}_{\beta \gamma}^{\alpha}$ are denoted respectively as ${ }^{(\gamma)} D$ and ${ }^{(\gamma)} \underline{D}$.

Curves on $U$ are parametrized

$$
u^{\alpha}=u^{\alpha}(\eta)=\left(x^{i}(\eta), y^{i}(\eta)\right), \eta_{1}<\eta<\eta_{2}
$$

where the corresponding tangent vector fields are defined

$$
v^{\alpha}=\frac{d u^{\alpha}}{d \eta}=\left(\frac{d x^{i}(\eta)}{d \eta}, \frac{d y^{j}(\eta)}{d \eta}\right)
$$

Definition 1 A curve $l$ is called auto parallel, a-parallel, on $V^{(n+m)}$ if its tangent vector field $v^{\alpha}$ satisfies the a-parallel equations

$$
v D v^{\alpha}=v^{\beta}(\gamma) D_{\beta} v^{\alpha}=\rho(\eta) v^{\alpha}
$$

where $\rho(\eta)$ is a scalar function on $V^{(n+m)}$.

Let a curve $\underline{l} \subset \underline{\xi}$ be given in parametric form $u^{\alpha}=u^{\alpha}(\eta), \eta_{1}<\eta<\eta_{2}$ with the tangent vector field $v^{\alpha}=\frac{d u^{\alpha}}{d \eta} \neq 0$. We suppose that a 2-dimensional distribution $E_{2}(\underline{l})$ is defined along $\underline{l}$, i.e. in every point $u \in \underline{l}$ a 2 -dimensional vector space $E_{2}(\underline{l}) \subset \underline{\xi}$ is fixed. The introduced distribution $E_{2}(\underline{l})$ is coplanar along $\underline{l}$ if every vector $\underline{p}^{\alpha}\left(u_{(0)}^{b}\right) \subset E_{2}(\underline{l}), u_{(0)}^{\beta} \subset \underline{l}$ rests contained in the same distribution after parallel transports along $\underline{l}$, i.e. $\underline{p}^{\alpha}\left(u^{\beta}(\eta)\right) \subset E_{2}(\underline{l})$.

Definition 2 A curve $\underline{l}$ is called nearly autoparallel, or, in brief, na-parallel, on the spacetime $\underline{V}^{(n+m)}$ if a coplanar along $\underline{l}$ distribution $E_{2}(\underline{l})$ containing the tangent to $\underline{l}$ vector field $v^{\alpha}(\eta)$, i.e. $v^{\alpha}(\eta) \subset E_{2}(\underline{l})$, is defined.

We can define nearly autoparallel maps of la-spacetimes as an anisotropic generalization of the constructions for the locally isotropic spaces (see ng- 32 and namaps 43, 40]):

Definition 3 Nearly autoparallel maps, na-maps, of la-spacetimes are defined as local 1-1 mappings $V^{(n+m)} \rightarrow \underline{V}^{(n+m)}$ which change every a-parallel on $V^{(n)}$ into a na-parallel on $\underline{V}^{(n+m)}$. 
Now we formulate the general conditions when some deformations (22) and (23) characterize na-maps:

Let an a-parallel $l \subset U$ is given by some functions $u^{\alpha}=u^{(\alpha)}(\eta), v^{\alpha}=\frac{d u^{\alpha}}{d \eta}, \eta_{1}<$ $\eta<\eta_{2}$, satisfying the equations (24). We suppose that to this a-parallel corresponds a na-parallel $\underline{l} \subset \underline{U}$ given by the same parameterization in a common for a chosen na-map coordinate system on $U$ and $\underline{U}$. This condition holds for the vectors $\underline{v}_{(1)}^{\alpha}=$ $v \underline{D} v^{\alpha}$ and $v_{(2)}^{\alpha}=v \underline{D} v_{(1)}^{\alpha}$ satisfying the equality

$$
\underline{v}_{(2)}^{\alpha}=\underline{a}(\eta) v^{\alpha}+\underline{b}(\eta) \underline{v}_{(1)}^{\alpha}
$$

for some scalar functions $\underline{a}(\eta)$ and $\underline{b}(\eta)$ (see Definitions 2 and 3). Putting the splittings (22) and (23) into the expressions for $\underline{v}_{(1)}^{\alpha}$ and $\underline{v}_{(2)}^{\alpha}$ from (25) we obtain:

$$
v^{\beta} v^{\gamma} v^{\delta}\left(D_{\beta} P_{. \gamma \delta}^{\alpha}+P_{. \beta \tau}^{\alpha} P_{. \gamma \delta}^{\tau}+Q_{. \beta \tau}^{\alpha} P_{. \gamma \delta}^{\tau}\right)=b v^{\gamma} v^{\delta} P_{. \gamma \delta}^{\alpha}+a v^{\alpha},
$$

where

$$
b(\eta, v)=\underline{b}-3 \rho, \quad \text { and } \quad a(\eta, v)=\underline{a}+\underline{b} \rho-v^{b} \partial_{b} \rho-\rho^{2}
$$

are called the deformation parameters of na-maps.

The algebraic equations for the deformation of torsion $Q_{. \beta \tau}^{\alpha}$ should be written as the compatibility conditions for a given nonmetricity tensor $\underline{K}_{\alpha \beta \gamma}$ on $\underline{V}^{(n+m)}$ ( or as the metricity conditions if the $\mathrm{d}$-connection $\underline{D}_{\alpha}$ is required to be metric):

$$
D_{\alpha} G_{\beta \gamma}-P_{. \alpha(\beta}^{\delta} G_{\gamma) \delta}-\underline{K}_{\alpha \beta \gamma}=Q_{. \alpha(\beta}^{\delta} G_{\gamma) \delta},
$$

where ( ) denotes the symmetrical alternation.

So, we have proved this

Theorem 1 The na-maps from a la-spacetime $V^{(n+m)}$ to la-spacetime $\underline{V}^{(n+m)}$ with a fixed common nonlinear connection structure, $N_{j}^{a}(u)=\underline{N}_{j}^{a}(u)$, and given $d-$ connections, $\Gamma_{. \beta \gamma}^{\alpha}$ on $V^{(n+m)}$ and $\underline{\Gamma}_{. \beta \gamma}^{\alpha}$ on $\underline{V}^{(n+m)}$, are locally parametrized by the solutions of equations (20) and (28) for every point $u^{\alpha}$ and direction $v^{\alpha}$ on $U \subset \underline{V}^{(n+m)}$.

We call (26) and (28) the basic equations for na-maps of la-spacetimes. They generalize the corresponding Sinyukov's equations [32] which were introduced for isotropic spaces provided with symmetric affine connection structure, hold for generalized Finsler metrics modeled on vector and tangent bundle spaces and consist a particular case of the na-maps of (super) vector bundles provided with $\mathrm{N}$-connection structures 40, 43].

\section{Classification of $\mathrm{Na}-\mathrm{Maps}$}

$\mathrm{Na}$-maps are classified on possible polynomial parametrizations on variables $v^{\alpha}$ of deformations parameters $a$ and $b$, see formulas (26) and (27)). 
Theorem 2 There are four classes of na-maps characterized by corresponding deformation parameters and tensors and basic equations:

1. for $n a_{(0)}-$ maps, $\pi_{(0)}-$ maps,

$$
P_{\beta \gamma}^{\alpha}(u)=\psi_{(\beta} \delta_{\gamma)}^{\alpha}
$$

$\left(\delta_{\beta}^{\alpha}\right.$ is Kronecker symbol and $\psi_{\beta}=\psi_{\beta}(u)$ is a covariant vector d-field);

2. for $n a_{(1)}-$ maps

$$
a(u, v)=a_{\alpha \beta}(u) v^{\alpha} v^{\beta}, \quad b(u, v)=b_{\alpha}(u) v^{\alpha}
$$

and $P_{. \beta \gamma}^{\alpha}(u)$ is the solution of equations

$$
D_{(\alpha} P_{. \beta \gamma)}^{\delta}+P_{(\alpha \beta}^{\tau} P_{. \gamma) \tau}^{\delta}-P_{(\alpha \beta}^{\tau} Q_{. \gamma) \tau}^{\delta}=b_{(\alpha} P_{. \beta \gamma)}^{\delta}+a_{(\alpha \beta} \delta_{\gamma)}^{\delta}
$$

3. for $n a_{(2)}-$ maps

$$
\begin{aligned}
a(u, v) & =a_{\beta}(u) v^{\beta}, \quad b(u, v)=\frac{b_{\alpha \beta} v^{\alpha} v^{\beta}}{\sigma_{\alpha}(u) v^{\alpha}}, \quad \sigma_{\alpha} v^{\alpha} \neq 0 \\
P_{. \alpha \beta}^{\tau}(u) & =\psi_{(\alpha} \delta_{\beta)}^{\tau}+\sigma_{(\alpha} F_{\beta)}^{\tau}
\end{aligned}
$$

and $F_{\beta}^{\alpha}(u)$ is the solution of equations

$$
D_{(\gamma} F_{\beta)}^{\alpha}+F_{\delta}^{\alpha} F_{(\gamma}^{\delta} \sigma_{\beta)}-Q_{. \tau(\beta}^{\alpha} F_{\gamma)}^{\tau}=\mu_{(\beta} F_{\gamma)}^{\alpha}+\nu_{(\beta} \delta_{\gamma)}^{\alpha}
$$

$\left(\mu_{\beta}(u), \nu_{\beta}(u), \psi_{\alpha}(u), \sigma_{\alpha}(u)\right.$ are covariant d-vectors);

4. for $n a_{(3)}-$ maps

$$
\begin{aligned}
b(u, v) & =\frac{\alpha_{\beta \gamma \delta} v^{\beta} v^{\gamma} v^{\delta}}{\sigma_{\alpha \beta} v^{\alpha} v^{\gamma}} \\
P_{. \beta \gamma}^{\alpha}(u) & =\psi_{(\beta} \delta_{\gamma)}^{\alpha}+\sigma_{\beta \gamma} \varphi^{\alpha}
\end{aligned}
$$

where $\varphi^{\alpha}$ is the solution of equations

$$
D_{\beta} \varphi^{\alpha}=\nu \delta_{\beta}^{\alpha}+\mu_{\beta} \varphi^{\alpha}+\varphi^{\gamma} Q_{. \gamma \delta}^{\alpha}
$$

$\alpha_{\beta \gamma \delta}(u), \sigma_{\alpha \beta}(u), \psi_{\beta}(u), \nu(u)$ and $\mu_{\beta}(u)$ are $d$-tensors.

Proof. We sketch the proof respectively for every point in the theorem:

1. It is easy to verify that a-parallel equations (24) on $V^{(n+m)}$ transform into similar ones on $\underline{V}^{(n+m)}$ if and only if deformations (22) with deformation dtensors of type $P^{\alpha}{ }_{\beta \gamma}(u)=\psi_{(\beta} \delta_{\gamma)}^{\alpha}$ are considered. 
2. Using corresponding to $n a_{(1)}$-maps parametrizations of $a(u, v)$ and $b(u, v)$ (see conditions of the theorem) for arbitrary $v^{\alpha} \neq 0$ on $U \in V^{(n+m)}$ and after a redefinition of deformation parameters we obtain that equations (29) hold if and only if $P_{\beta \gamma}^{\alpha}$ satisfies (22).

3. In a similar manner we obtain basic $n a_{(2)}$-map equations (31) from (26) by considering $n a_{(2)}$-parametrizations of deformation parameters and d-tensor.

4. For $n a_{(3)}$-maps we mast take into consideration deformations of torsion (23) and introduce $n a_{(3)}$-parametrizations for $b(u, v)$ and $P^{\alpha}{ }_{\beta \gamma}$ into the basic naequations (26). The resulting equations, for $n a_{(3)}-$ maps, are equivalent to equations (33) (with a corresponding redefinition of deformation parameters).

We point out that for $\pi_{(0)}$-maps we have not differential equations on $P_{. \beta \gamma}^{\alpha}$ (in the isotropic case one considers a first order system of differential equations on metric [32]; we omit constructions with deformation of metric in this Section).

To formulate invariant conditions for reciprocal na-maps (when every a-parallel on $\underline{V}^{(n+m)}$ is also transformed into na-parallel on $\left.V^{(n+m)}\right)$ it is convenient to introduce into consideration the curvature and Ricci tensors defined for auxiliary connection $\gamma_{. \beta \gamma}^{\alpha}$ :

$$
r_{\alpha . \beta \tau}^{. \delta}=\partial_{[\beta} \gamma_{. \tau] \alpha}^{\delta}+\gamma_{. \rho[\beta}^{\delta} \gamma_{. \tau] \alpha}^{\rho}+\gamma_{\alpha \phi}^{\delta} w_{\beta \tau}^{\phi}
$$

and, respectively, $r_{\alpha \tau}=r_{\alpha . \gamma \tau}^{. \gamma}$, where [ ] denotes antisymmetric alternation of indices, and to define values:

$$
\begin{aligned}
& { }^{(0)} T_{. \alpha \beta}^{\mu}=\Gamma_{. \alpha \beta}^{\mu}-T_{. \alpha \beta}^{\mu}-\frac{1}{(n+m+1)}\left(\delta_{(\alpha}^{\mu} \Gamma_{. \beta) \delta}^{\delta}-\delta_{(\alpha}^{\mu} T_{. \beta) \gamma}^{\delta}\right), \\
& { }^{(0)} W_{\alpha \cdot \beta \gamma}^{\cdot \tau}=r_{\alpha \cdot \beta \gamma}^{\cdot \tau}+\frac{1}{n+m+1}\left[\gamma_{\cdot \varphi \tau}^{\tau} \delta_{(\alpha}^{\tau} w_{\beta) \gamma}^{\varphi}-\left(\delta_{\alpha}^{\tau} r_{[\gamma \beta]}+\delta_{\gamma}^{\tau} r_{[\alpha \beta]}-\delta_{\beta}^{\tau} r_{[\alpha \gamma]}\right)\right] \\
& -\frac{1}{(n+m+1)^{2}}\left[\delta_{\alpha}^{\tau}\left(2 \gamma_{\cdot \varphi \tau}^{\tau} w^{\varphi}{ }_{[\gamma \beta]}-\gamma_{\cdot \tau[\gamma}^{\tau} w_{\beta] \varphi}^{\varphi}\right)\right. \\
& \left.+\delta_{\gamma}^{\tau}\left(2 \gamma_{\cdot{ }^{\prime} \tau}^{\tau} w_{\alpha \beta}^{\varphi}-\gamma_{\cdot \alpha \tau}^{\tau} w_{\beta \varphi}^{\varphi}\right)-\delta_{\beta}^{\tau}\left(2 \gamma_{\cdot \varphi \tau}^{\tau} w_{\alpha \gamma}^{\varphi}-\gamma_{\cdot \alpha \tau}^{\tau} w_{\gamma \varphi}^{\varphi}\right)\right] \text {, } \\
& { }^{(3)} T_{. \alpha \beta}^{\delta}=\gamma_{. \alpha \beta}^{\delta}+\frac{1}{n+m}\left(\delta_{\alpha}^{\gamma}-\epsilon \varphi^{\delta} q_{\alpha}\right)\left[\gamma_{. \beta \tau}^{\tau}+\epsilon \varphi^{\tau(\gamma)} D_{\beta} q_{\tau}\right. \\
& \left.+\frac{1}{n+m-1} q_{\beta}\left(\epsilon \varphi^{\tau} \gamma_{. \tau \lambda}^{\lambda}+\varphi^{\lambda} \varphi^{\tau(\gamma)} D_{\tau} q_{\lambda}\right)\right]+\epsilon \varphi^{\tau(\gamma)} D_{\beta} q_{\tau} \\
& -\frac{1}{n+m}\left(\delta_{\beta}^{\delta}-\epsilon \varphi^{\delta} q_{\beta}\right)\left[\gamma_{. \alpha \tau}^{\tau}+\epsilon \varphi^{\tau(\gamma)} D_{\alpha} q_{\tau}\right. \\
& \left.+\frac{1}{n+m-1} q_{\alpha}\left(\epsilon \varphi^{\tau} \gamma_{. \tau \lambda}^{\lambda}+\varphi^{\lambda} \varphi^{\tau(\gamma)} D_{\tau} q_{\lambda}\right)\right] \\
& { }^{(3)} W^{\alpha} \cdot \beta \gamma \delta=\rho_{\beta . \gamma \delta}^{. \alpha}+\epsilon \varphi^{\alpha} q_{\tau} \rho_{\beta . \gamma \delta}^{\cdot \tau} \\
& +\left(\delta_{\delta}^{\alpha}-\epsilon \varphi^{\alpha} q_{\delta}\right) p_{\beta \gamma}-\left(\delta_{\gamma}^{\alpha}-\epsilon \varphi^{\alpha} q_{\gamma}\right) p_{\beta \delta}-\left(\delta_{\beta}^{\alpha}-\epsilon \varphi^{\alpha} q_{\beta}\right) p_{[\gamma \delta]},
\end{aligned}
$$




$$
\begin{aligned}
(n+m-2) p_{\alpha \beta}= & -\rho_{\alpha \beta}-\epsilon q_{\tau} \varphi^{\gamma} \rho_{\alpha . \beta \gamma}^{. \tau}+\frac{1}{n+m}\left[\rho_{\tau . \beta \alpha}^{\cdot \tau}-\epsilon q_{\tau} \varphi^{\gamma} \rho_{\gamma . \beta \alpha}^{\cdot \tau}+\epsilon q_{\beta} \varphi^{\tau} \rho_{\alpha \tau}\right. \\
& \left.+\epsilon q_{\alpha}\left(-\varphi^{\gamma} \rho_{\tau . \beta \gamma}^{\cdot \tau}+\epsilon q_{\tau} \varphi^{\gamma} \varphi^{\delta} \rho_{\gamma . \beta \delta}^{\cdot \tau}\right]\right),
\end{aligned}
$$

where $q_{\alpha} \varphi^{\alpha}=\epsilon= \pm 1$

$$
\rho_{\beta \gamma \delta}^{\alpha}=r_{\beta \cdot \gamma \delta}^{\cdot \alpha}+\frac{1}{2}\left(\psi_{(\beta} \delta_{\varphi)}^{\alpha}+\sigma_{\beta \varphi} \varphi^{\tau}\right) w_{\gamma \delta}^{\varphi}
$$

and $\rho_{\alpha \beta}=\rho_{\cdot \alpha \beta \tau}^{\tau}$.

For similar values on $\underline{V}^{(n+m)}$ we write, for instance,

$$
\left.\underline{\rho}_{\cdot \beta \gamma \delta}^{\alpha}=\underline{r}_{\beta \cdot \gamma \delta}^{\cdot \alpha}-\frac{1}{2}\left(\psi_{(\beta} \delta_{\varphi)}^{\alpha}-\sigma_{\beta \varphi} \varphi^{\tau}\right) w_{\gamma \delta}^{\varphi}\right)
$$

and note that ${ }^{(0)} \underline{T}_{. \beta \gamma}^{\alpha},{ }^{(0)} \underline{W}_{. \alpha \beta \gamma}^{\nu}, \hat{T}_{. \beta \gamma}^{\alpha}, \check{T}_{. \beta \tau}^{\alpha}, \hat{W}_{. \alpha \beta \gamma}^{\delta}, \check{W}_{. \alpha \beta \gamma}^{\delta},{ }^{(3)} \underline{T}_{. \alpha \beta}^{\delta},{ }^{(3)} \underline{W}_{. \beta \gamma \delta}^{\alpha}$ are given, correspondingly, by auxiliary connections $\underline{\Gamma}_{. \alpha \beta}^{\mu}$,

$$
\begin{gathered}
\star \gamma_{. \beta \lambda}^{\alpha}=\gamma_{. \beta \lambda}^{\alpha}+\epsilon F_{\tau}^{\alpha(\gamma)} D_{(\beta} F_{\lambda)}^{\tau}, \quad \check{\gamma}_{. \beta \lambda}^{\alpha}=\widetilde{\gamma}_{. \beta \lambda}^{\alpha}+\epsilon F_{\tau}^{\lambda} \widetilde{D}_{(\beta} F_{\lambda)}^{\tau}, \\
\widetilde{\gamma}_{. \beta \tau}^{\alpha}=\gamma_{. \beta \tau}^{\alpha}+\sigma_{(\beta} F_{\tau)}^{\alpha}, \quad \hat{\gamma}_{. \beta \lambda}^{\alpha}=\star \gamma_{. \beta \lambda}^{\alpha}+\widetilde{\sigma}_{(\beta} \delta_{\lambda)}^{\alpha},
\end{gathered}
$$

where $\tilde{\sigma}_{\beta}=\sigma_{\alpha} F_{\beta}^{\alpha}$.

Theorem 3 Four classes of reciprocal na-maps of la-spacetimes are characterized by corresponding invariant criterions:

1. for a-maps

$$
\begin{aligned}
{ }^{(0)} T_{. \alpha \beta}^{\mu} & ={ }^{{ }^{(0)}} \underline{T}_{. \alpha \beta}^{\mu}, \\
{ }^{(0)} W_{. \alpha \beta \gamma}^{\delta} & ={ }^{(0)} \underline{W}_{. \alpha \beta \gamma}^{\delta} ;
\end{aligned}
$$

2. for $n a_{(1)}-$ maps

$$
\begin{aligned}
3\left({ }^{(\gamma)} D_{\lambda} P_{. \alpha \beta}^{\delta}+\right. & \left.P_{. \tau \lambda}^{\delta} P_{. \alpha \beta}^{\tau}\right)=r_{(\alpha . \beta) \lambda}^{. \delta}-\underline{r}_{(\alpha . \beta) \lambda}^{. \delta} \\
& +\left[T_{. \tau(\alpha}^{\delta} P_{. \beta \lambda)}^{\tau}+Q_{. \tau(\alpha}^{\delta} P_{. \beta \lambda)}^{\tau}+b_{(\alpha} P_{. \beta \lambda)}^{\delta}+\delta_{(\alpha}^{\delta} a_{\beta \lambda)}\right]
\end{aligned}
$$

3. for $n a_{(2)}-$ maps

$$
\begin{aligned}
\hat{T}_{. \beta \tau}^{\alpha} & =\star T_{. \beta \tau}^{\alpha}, \\
\hat{W}_{. \alpha \beta \gamma}^{\delta} & =\star W_{. \alpha \beta \gamma}^{\delta} ;
\end{aligned}
$$

4. for $n a_{(3)}-$ maps

$$
\begin{aligned}
{ }^{(3)} T_{. \beta \gamma}^{\alpha} & ={ }^{(3)} \underline{T}_{. \beta \gamma}^{\alpha}, \\
{ }^{(3)} W_{. \beta \gamma \delta}^{\alpha} & ={ }^{(3)} \underline{W}_{. \beta \gamma \delta}^{\alpha} .
\end{aligned}
$$




\section{Proof.}

1. Let us prove that the a-invariant conditions (34) hold. Deformations of $\mathrm{d}-$ connections of type

$$
{ }^{(0)} \underline{\gamma}_{\cdot \alpha \beta}^{\mu}=\gamma_{\alpha \beta}^{\mu}+\psi_{(\alpha} \delta_{\beta)}^{\mu}
$$

define a-applications. Contracting indices $\mu$ and $\beta$ we can write

$$
\psi_{\alpha}=\frac{1}{m+n+1}\left(\underline{\gamma}_{\alpha \beta}^{\beta}-\gamma_{\alpha \beta}^{\beta}\right)
$$

Introducing the $\mathrm{d}-$-vector $\psi_{\alpha}$ into previous relation and expressing

$$
\gamma_{\beta \tau}^{\alpha}=-T_{\beta \tau}^{\alpha}+\Gamma_{\beta \tau}^{\alpha}
$$

and similarly for underlined values we obtain the first invariant conditions from (34).

Putting deformation (38) into the formulas for $\underline{r}_{\alpha \cdot \beta \gamma}^{\cdot \tau}$ and $\underline{r}_{\alpha \beta}=\underline{r}_{\alpha \tau \beta \tau}^{\cdot \tau}$ we obtain respectively the relations

$$
\underline{r}_{\alpha \cdot \beta \gamma}^{\cdot \tau}-r_{\alpha \cdot \beta \gamma}^{\cdot \tau}=\delta_{\alpha}^{\tau} \psi_{[\gamma \beta]}+\psi_{\alpha[\beta} \delta_{\gamma]}^{\tau}+\delta_{(\alpha}^{\tau} \psi_{\varphi)} w_{\beta \gamma}^{\varphi}
$$

and

$$
\underline{r}_{\alpha \beta}-r_{\alpha \beta}=\psi_{[\alpha \beta]}+(n+m-1) \psi_{\alpha \beta}+\psi_{\varphi} w_{\beta \alpha}^{\varphi}+\psi_{\alpha} w_{\beta \varphi}^{\varphi},
$$

where

$$
\psi_{\alpha \beta}={ }^{(\gamma)} D_{\beta} \psi_{\alpha}-\psi_{\alpha} \psi_{\beta}
$$

Putting (38) into (40) and (41) we can express $\psi_{[\alpha \beta]}$ as

$$
\begin{gathered}
\psi_{[\alpha \beta]}=\frac{1}{n+m+1}\left[\underline{r}_{[\alpha \beta]}+\frac{2}{n+m+1} \underline{\gamma}_{\cdot \varphi \tau}^{\tau} w_{[\alpha \beta]}^{\varphi}-\frac{1}{n+m+1} \underline{\gamma}_{\cdot \tau[\alpha}^{\tau} w_{\beta] \varphi}^{\varphi}\right] \\
-\frac{1}{n+m+1}\left[r_{[\alpha \beta]}+\frac{2}{n+m+1} \gamma_{\varphi \tau}^{\tau} w_{[\alpha \beta]}^{\varphi}-\frac{1}{n+m+1} \gamma_{\tau[\alpha}^{\tau} w_{\beta] \varphi}^{\varphi}\right] .
\end{gathered}
$$

To simplify our consideration we can choose an a-transform, parametrized by corresponding $\psi$-vector from (38), (or fix a local coordinate cart) the antisymmetrized relations (38) to be satisfied by d-tensor

$$
\begin{aligned}
\psi_{\alpha \beta}= & \frac{1}{n+m+1}\left[\underline{r}_{\alpha \beta}+\frac{2}{n+m+1} \underline{\gamma}_{\cdot \varphi \tau}^{\tau} w_{\alpha \beta}^{\varphi}-\frac{1}{n+m+1} \underline{\gamma}_{\cdot \alpha \tau}^{\tau} w_{\beta \varphi}^{\varphi}\right. \\
& \left.-r_{\alpha \beta}-\frac{2}{n+m+1} \gamma_{\varphi \tau}^{\tau} w_{\alpha \beta}^{\varphi}+\frac{1}{n+m+1} \gamma_{\alpha \tau}^{\tau} w_{\beta \varphi}^{\varphi}\right]
\end{aligned}
$$

Introducing expressions (38), (42) and (43) into deformation of curvature (39) we obtain the second condition from (34) of a-map invariance:

$$
{ }^{(0)} W_{\alpha \cdot \beta \gamma}^{\cdot \delta}={ }^{(0)} \underline{W}_{\alpha \cdot \beta \gamma}^{\cdot \delta},
$$


where the Weyl d-tensor on $\underline{V}^{(n+m)}$ is defined as

$$
\begin{aligned}
{ }^{(0)} \underline{W}_{\alpha \cdot \beta \gamma}^{\cdot \tau}= & \underline{r}_{\alpha \cdot \beta \gamma}^{\cdot \tau}+\frac{1}{n+m+1}\left[\underline{\gamma}_{\cdot \varphi \tau}^{\tau} \delta_{(\alpha}^{\tau} w_{\beta, \gamma}^{\varphi}-\left(\delta_{\alpha}^{\tau} \underline{r}_{[\gamma \beta]}+\delta_{\gamma}^{\tau} \underline{r}_{[\alpha \beta]}-\delta_{\beta}^{\tau} \underline{r}_{[\alpha \gamma]}\right)\right] \\
& -\frac{1}{(n+m+1)^{2}}\left[\delta_{\alpha}^{\tau}\left(2 \underline{\gamma}_{\cdot \varphi \tau}^{\tau} w^{\varphi}{ }_{[\gamma \beta]}-\underline{\gamma}_{\cdot \tau[\gamma}^{\tau} w_{\beta] \varphi}^{\varphi}\right)\right. \\
& \left.+\delta_{\gamma}^{\tau}\left(2 \underline{\gamma}_{\cdot \varphi \tau}^{\tau} w_{\alpha \beta}^{\varphi}-\underline{\gamma}_{\cdot \alpha \tau}^{\tau} w_{\beta \varphi}^{\varphi}\right)-\delta_{\beta}^{\tau}\left(2 \underline{\gamma}_{\cdot \varphi \tau}^{\tau} w_{\alpha \gamma}^{\varphi}-\underline{\gamma}_{\cdot \alpha \tau}^{\tau} w_{\gamma \varphi}^{\varphi}\right)\right] .
\end{aligned}
$$

2. To obtain $n a_{(1)}$-invariant conditions we rewrite $n a_{(1)}$-equations (29) as to consider in explicit form covariant derivation ${ }^{(\gamma)} D$ and deformations (22) and (23):

$$
\begin{gathered}
2{ }^{(\gamma)} D_{\alpha} P_{\beta \gamma}^{\delta}+{ }^{(\gamma)} D_{\beta} P^{\delta}{ }_{\alpha \gamma}+{ }^{(\gamma)} D_{\gamma} P_{\alpha \beta}^{\delta}+P_{\tau \alpha}^{\delta} P_{\beta \gamma}^{\tau}+P_{\tau \beta}^{\delta} P_{\alpha \gamma}^{\tau} \\
\left.+P^{\delta}{ }_{\tau \gamma} P^{\tau}{ }_{\alpha \beta}\right)=T^{\delta}{ }_{\tau(\alpha} P^{\tau}{ }_{\beta \gamma)}+H^{\delta}{ }_{\tau(\alpha} P^{\tau}{ }_{\beta \gamma)}+b_{(\alpha} P_{\beta \gamma)}^{\delta}+a_{(\alpha \beta} \delta_{\gamma)}^{\delta} .
\end{gathered}
$$

Alternating the first two indices in (44) we have

$$
\begin{aligned}
2\left(\underline{r}_{(\alpha \cdot \beta) \gamma}^{\delta}-r_{(\alpha \cdot \beta) \gamma}^{\cdot \delta}\right)= & 2\left({ }^{(\gamma)} D_{\alpha} P_{\beta \gamma}^{\delta}+{ }_{\beta \gamma}^{(\gamma)} D_{\beta} P_{\alpha \gamma}^{\delta}-2^{(\gamma)} D_{\gamma} P_{\alpha \beta}^{\delta}\right. \\
& \left.+P^{\delta}{ }_{\tau \alpha} P^{\tau}{ }_{\beta \gamma}+P^{\delta}{ }_{\tau \beta} P^{\tau}{ }_{\alpha \gamma}-2 P^{\delta}{ }_{\tau \gamma} P^{\tau}{ }_{\alpha \beta}\right) .
\end{aligned}
$$

Substituting the last expression from (44) and rescalling the deformation parameters and d-tensors we obtain the conditions (29).

3. Now we prove the invariant conditions for $n a_{(0)}$-maps satisfying conditions

$$
\epsilon \neq 0 \text { and } \epsilon-F_{\beta}^{\alpha} F_{\alpha}^{\beta} \neq 0
$$

Let define the auxiliary $\mathrm{d}$-connection

$$
\tilde{\gamma}_{\cdot \beta \tau}^{\alpha}=\underline{\gamma}_{\cdot \beta \tau}^{\alpha}-\psi_{(\beta} \delta_{\tau)}^{\alpha}=\gamma_{\beta \tau}^{\alpha}+\sigma_{(\beta} F_{\tau)}^{\alpha}
$$

and write

$$
\tilde{D}_{\gamma}={ }^{(\gamma)} D_{\gamma} F_{\beta}^{\alpha}+\tilde{\sigma}_{\gamma} F_{\beta}^{\alpha}-\epsilon \sigma_{\beta} \delta_{\gamma}^{\alpha},
$$

where $\tilde{\sigma}_{\beta}=\sigma_{\alpha} F_{\beta}^{\alpha}$, or, as a consequence from the last equality,

$$
\sigma_{(\alpha} F_{\beta)}^{\tau}=\epsilon F_{\lambda}^{\tau}\left({ }^{(\gamma)} D_{(\alpha} F_{\beta)}^{\alpha}-\tilde{D}_{(\alpha} F_{\beta)}^{\lambda}\right)+\tilde{\sigma}_{(} \alpha \delta_{\beta)}^{\tau} .
$$

Introducing the auxiliary connections

$$
\star \gamma_{\cdot \beta \lambda}^{\alpha}=\gamma_{\cdot \beta \lambda}^{\alpha}+\epsilon F_{\tau}^{\alpha(\gamma)} D_{(\beta} F_{\lambda)}^{\tau}, \check{\gamma}_{\cdot \beta \lambda}^{\alpha}=\tilde{\gamma}_{\cdot \beta \lambda}^{\alpha}+\epsilon F_{\tau}^{\alpha} \tilde{D}_{(\beta} F_{\lambda)}^{\tau}
$$

we can express the deformation (45) in a form characteristic for a-maps:

$$
\hat{\gamma}_{\cdot \beta \gamma}^{\alpha}=\star \gamma_{\cdot \beta \gamma}^{\alpha}+\tilde{\sigma}_{(\beta} \delta_{\lambda)}^{\alpha} \text {. }
$$

Now it's obvious that $n a_{(2)}$-invariant conditions (46) are equivalent with ainvariant conditions (34) written for $\mathrm{d}$-connection (46). As a matter of principle we can write formulas for such $n a_{(2)}$-invariants in terms of "underlined" and "non-underlined" values by expressing consequently all used auxiliary connections as deformations of "prime" connections on $V^{(n+m)}$ and "final" connections on $\underline{V}^{(n+m)}$. We omit such tedious calculations in this work. 
4. Finally, we prove the last statement, for $n a_{(3)}-$ maps, of this theorem. Let

$$
q_{\alpha} \varphi^{\alpha}=e= \pm 1
$$

where $\varphi^{\alpha}$ is contained in

$$
\underline{\gamma}_{\cdot \beta \gamma}^{\alpha}=\gamma_{\beta \gamma}^{\alpha}+\psi_{(\beta} \delta_{\gamma)}^{\alpha}+\sigma_{\beta \gamma} \varphi^{\alpha}
$$

Acting with operator ${ }^{(\gamma)} \underline{D}_{\beta}$ on (46) we write

$$
{ }^{(\gamma)} \underline{D}_{\beta} q_{\alpha}={ }^{(\gamma)} D_{\beta} q_{\alpha}-\psi_{(\alpha} q_{\beta)}-e \sigma_{\alpha \beta}
$$

Contracting (48) with $\varphi^{\alpha}$ we can express

$$
e \varphi^{\alpha} \sigma_{\alpha \beta}=\varphi^{\alpha}\left({ }^{(\gamma)} D_{\beta} q_{\alpha}-{ }^{(\gamma)} \underline{D}_{\beta} q_{\alpha}\right)-\varphi_{\alpha} q^{\alpha} q_{\beta}-e \psi_{\beta}
$$

Putting the last formula in (47) contracted on indices $\alpha$ and $\gamma$ we obtain

$$
\left.(n+m) \psi_{\beta}=\underline{\gamma}_{\cdot \alpha \beta}^{\alpha}-\gamma_{\alpha \beta}^{\alpha}+e \psi_{\alpha} \varphi^{\alpha} q_{\beta}+e \varphi^{\alpha} \varphi^{\beta}{ }^{(\gamma)} \underline{D}_{\beta}-{ }^{(\gamma)} D_{\beta}\right) .
$$

¿From these relations, taking into consideration (46), we have

$$
(n+m-1) \psi_{\alpha} \varphi^{\alpha}=\varphi^{\alpha}\left(\underline{\gamma}_{\cdot \alpha \beta}^{\alpha}-\gamma_{\alpha \beta}^{\alpha}\right)+e \varphi^{\alpha} \varphi^{\beta}\left({ }^{(\gamma)} \underline{D}_{\beta} q_{\alpha}-{ }^{(\gamma)} D_{\beta} q_{\alpha}\right)
$$

Using the equalities and identities (48) and (49) we can express the deformations (47) as the first $n a_{(3)}$-invariant conditions from (37).

To prove the second class of $n a_{(3)}$-invariant conditions we introduce two additional d-tensors:

$$
\rho_{\beta \gamma \delta}^{\alpha}=r_{\beta \cdot \gamma \delta}^{\cdot \alpha}+\frac{1}{2}\left(\psi_{(\beta} \delta_{\varphi)}^{\alpha}+\sigma_{\beta \varphi} \varphi^{\tau}\right) w_{\gamma \delta}^{\varphi}
$$

and

$$
\underline{\rho}_{\cdot \beta \gamma \delta}^{\alpha}=\underline{r}_{\beta \cdot \gamma \delta}^{\cdot \alpha}-\frac{1}{2}\left(\psi_{(\beta} \delta_{\varphi)}^{\alpha}-\sigma_{\beta \varphi} \varphi^{\tau}\right) w_{\gamma \delta}^{\varphi} .
$$

Using deformation (47) and (50) we write relation

$$
\tilde{\sigma}_{\cdot \beta \gamma \delta}^{\alpha}=\underline{\rho}_{\cdot \beta \gamma \delta}^{\alpha}-\rho_{\cdot \beta \gamma \delta}^{\alpha}=\psi_{\beta[\delta} \delta_{\gamma]}^{\alpha}-\psi_{[\gamma \delta]} \delta_{\beta}^{\alpha}-\sigma_{\beta \gamma \delta} \varphi^{\alpha}
$$

where

$$
\psi_{\alpha \beta}={ }^{(\gamma)} D_{\beta} \psi_{\alpha}+\psi_{\alpha} \psi_{\beta}-\left(\nu+\varphi^{\tau} \psi_{\tau}\right) \sigma_{\alpha \beta}
$$

and

$$
\sigma_{\alpha \beta \gamma}={ }^{(\gamma)} D_{[\gamma} \sigma_{\beta] \alpha}+\mu_{[\gamma} \sigma_{\beta] \alpha}-\sigma_{\alpha[\gamma} \sigma_{\beta] \tau} \varphi^{\tau}
$$

Let multiply (51) on $q_{\alpha}$ and write (taking into account relations (46)) the relation

$$
e \sigma_{\alpha \beta \gamma}=-q_{\tau} \tilde{\sigma}_{\cdot \alpha \beta \delta}^{\tau}+\psi_{\alpha[\beta} q_{\gamma]}-\psi_{[\beta \gamma]} q_{\alpha} .
$$


The next step is to express $\psi_{\alpha \beta}$ trough d-objects on $V^{(n+m)}$. To do this we contract indices $\alpha$ and $\beta$ in (51) and obtain

$$
(n+m) \psi_{[\alpha \beta]}=-\sigma_{\cdot \tau \alpha \beta}^{\tau}+e q_{\tau} \varphi^{\lambda} \sigma_{\cdot \lambda \alpha \beta}^{\tau}-e \tilde{\psi}_{[\alpha} \tilde{\psi}_{\beta]} .
$$

Then contracting indices $\alpha$ and $\delta$ in (51) and using (52) we write

$$
(n+m-2) \psi_{\alpha \beta}=\tilde{\sigma}_{\cdot \alpha \beta \tau}^{\tau}-e q_{\tau} \varphi^{\lambda} \tilde{\sigma}_{\cdot \alpha \beta \lambda}^{\tau}+\psi_{[\beta \alpha]}+e\left(\tilde{\psi}_{\beta} q_{\alpha}-\hat{\psi}_{(\alpha} q_{\beta)}\right),
$$

where $\hat{\psi}_{\alpha}=\varphi^{\tau} \psi_{\alpha \tau}$. If the both parts of (53) are contracted with $\varphi^{\alpha}$, it results that

$$
(n+m-2) \tilde{\psi}_{\alpha}=\varphi^{\tau} \sigma_{\cdot \tau \alpha \lambda}^{\lambda}-e q_{\tau} \varphi^{\lambda} \varphi^{\delta} \sigma_{\lambda \alpha \delta}^{\tau}-e q_{\alpha},
$$

and, in consequence of $\sigma_{\beta(\gamma \delta)}^{\alpha}=0$, we have

$$
(n+m-1) \varphi=\varphi^{\beta} \varphi^{\gamma} \sigma_{\cdot \beta \gamma \alpha}^{\alpha} .
$$

By using the last expressions we can write

$$
(n+m-2) \underline{\psi}_{\alpha}=\varphi^{\tau} \sigma_{\cdot \tau \alpha \lambda}^{\lambda}-e q_{\tau} \varphi^{\lambda} \varphi^{\delta} \sigma_{\cdot \lambda \alpha \delta}^{\tau}-e(n+m-1)^{-1} q_{\alpha} \varphi^{\tau} \varphi^{\lambda} \sigma_{\cdot \tau \lambda \delta}^{\delta} .
$$

Contracting (53) with $\varphi^{\beta}$ we have

$$
(n+m) \hat{\psi}_{\alpha}=\varphi^{\tau} \sigma_{\cdot \alpha \tau \lambda}^{\lambda}+\tilde{\psi}_{\alpha}
$$

and taking into consideration (54) we can express $\hat{\psi}_{\alpha}$ through $\sigma_{\cdot \beta \gamma \delta}^{\alpha}$.

As a consequence of (52)-(54) we obtain this formulas for d-tensor $\psi_{\alpha \beta}$ :

$$
\begin{aligned}
& (n+m-2) \psi_{\alpha \beta}=\sigma_{\cdot \alpha \beta \tau}^{\tau}-e q_{\tau} \varphi^{\lambda} \sigma_{\cdot \alpha \beta \lambda}^{\tau} \\
& +\frac{1}{n+m}\left\{-\sigma_{\cdot \tau \beta \alpha}^{\tau}+e q_{\tau} \varphi^{\lambda} \sigma_{\cdot \lambda \beta \alpha}^{\tau}-q_{\beta}\left(e \varphi^{\tau} \sigma_{\cdot \alpha \tau \lambda}^{\lambda}-q_{\tau} \varphi^{\lambda} \varphi^{\delta} \sigma_{\cdot \alpha \lambda \delta}^{\tau}\right)+e q_{\alpha} \times\right. \\
& \left.\left[\varphi^{\lambda} \sigma_{\cdot \tau \beta \lambda}^{\tau}-e q_{\tau} \varphi^{\lambda} \varphi^{\delta} \sigma_{\cdot \lambda \beta \delta}^{\tau}-\frac{e}{n+m-1} q_{\beta}\left(\varphi^{\tau} \varphi^{\lambda} \sigma_{\cdot \tau \gamma \delta}^{\delta}-e q_{\tau} \varphi^{\lambda} \varphi^{\delta} \varphi^{\varepsilon} \sigma_{\cdot \lambda \delta \varepsilon}^{\tau}\right)\right]\right\} .
\end{aligned}
$$

Finally, putting the last formula and (52) into (51) and after a rearrangement of terms we obtain the second group of $n a_{(3)}$-invariant conditions (37). If necessary we can rewrite these conditions in terms of geometrical objects on $V^{(n+m)}$ and $\underline{V}^{(n+m)}$. To do this we mast introduce splittings (50) into (37).

For the particular case of $n a_{(3)}$-maps when $\psi_{\alpha}=0, \varphi_{\alpha}=g_{\alpha \beta} \varphi^{\beta}=\frac{\delta}{\delta u^{\alpha}}(\ln \Omega)$, $\Omega(u)>0$ and $\sigma_{\alpha \beta}=g_{\alpha \beta}$ we define a subclass of conformal transforms $\underline{g}_{\alpha \beta}(u)=$ $\Omega^{2}(u) g_{\alpha \beta}$ which, in consequence of the fact that d-vector $\varphi_{\alpha}$ must satisfy equations (33), generalizes the class of concircular transforms (see [32] for references and details on concircular mappings of Riemannaian spaces).

We emphasize that the basic na-equations (29)-(33) are systems of first order partial differential equations. The study of their geometrical properties and definition of integral varieties, general and particular solutions are possible by using the 
formalism of Pffaf systems [12, 43, 40]. Here we point out that by using algebraic methods we can always verify if systems of na-equations of type (29)-(33) are, or not, involute, even to find their explicit solutions it is a difficult task (see more detailed considerations for isotropic ng-maps in [32 and, on language of Pffaf systems for na-maps, in 40, 43]). We can also formulate the Cauchy problem for na-equations on $V^{(n+m)}$ and choose deformation parameters (27) as to make involute mentioned equations for the case of maps to a given background space $\underline{V}^{(n+m)}$. If a solution, for example, of $n a_{(1)}$-map equations exists, we say that the la-spacetime $V^{(n+m)}$ is $n a_{(1)}$-projective to the la-spacetime $\underline{V}^{(n+m)}$. In general, we have to introduce chains of na-maps in order to obtain involute systems of equations for maps (superpositions of na-maps) from $V^{(n+m)}$ to $\underline{V}^{(n+m)}$ :

$$
U \stackrel{n g<i_{1}>}{\longrightarrow} U_{\underline{1}} \stackrel{n g<i_{2}>}{\longrightarrow} \ldots \stackrel{n g<i_{k-1}>}{\longrightarrow} U_{\underline{k-1}} \stackrel{n g<i_{k}>}{\longrightarrow} \underline{U}
$$

where

$$
U \subset V^{(n+m)}, U_{\underline{1}} \subset V^{(n+m)}{ }_{\underline{1}}, \ldots, U_{\underline{k-1}} \subset V^{(n+m)}{ }_{\underline{k-1}}, U_{\underline{k}} \subset V^{(n+m)}{ }_{\underline{k}}, \underline{U} \subset V^{(n+m)}
$$

with corresponding splittings of auxiliary symmetric connections

$$
\underline{\gamma}_{. \beta \gamma}^{\alpha}={<i i_{1}>}_{. \beta \gamma}^{\alpha}+_{<i_{2}>} P_{. \beta \gamma}^{\alpha}+\cdots++_{<i_{k}>} P_{. \beta \gamma}^{\alpha}
$$

and torsion

$$
\underline{T}_{. \beta \gamma}^{\alpha}=T_{. \beta \gamma}^{\alpha}+_{<i_{1}>} Q_{. \beta \gamma}^{\alpha}+_{<i_{2}>} Q_{. \beta \gamma}^{\alpha}+\cdots+_{<i_{k}>} Q_{. \beta \gamma}^{\alpha}
$$

where the indices $<i_{1}>=0,1,2,3$, denote possible types of na-maps.

Definition 4 A la-spacetime $V^{(n+m)}$ is nearly conformally projective to the laspacetime $\underline{V}^{(n+m)}, n c: V^{(n+m)} \rightarrow \underline{V}^{(n+m)}$, if there is a finite chain of na-maps from $V^{(n+m)}$ to $\underline{V}^{(n+m)}$.

For nearly conformal maps we formulate:

Theorem 4 For every fixed triples $\left(N_{j}^{a}, \Gamma_{. \beta \gamma}^{\alpha}, U \subset V^{(n+m)}\right)$ and $\left(N_{j}^{a}, \underline{\Gamma}_{. \beta \gamma}^{\alpha}, \underline{U} \subset\right.$ $\left.\underline{V}^{(n+m)}\right)$ and given components of nonlinear connection, $d$-connection and $d$-metric being of class $C^{r}(U), C^{r}(\underline{U}), r>3$, there is a finite chain of na-maps $n c: U \rightarrow \underline{U}$.

The proof is to performed by introducing a finite number of na-maps with corresponding components of deformation parameters and deformation tensors in order to transform step by step the coefficients of d-connection $\Gamma_{\gamma \delta}^{\alpha}$ into the $\left.\underline{\Gamma}_{\beta \gamma}^{\alpha}\right)$.

Now we introduce the concept of the Category of la-spacetimes, $\mathcal{C}\left(V^{(n+m)}\right)$. The elements of $\mathcal{C}\left(V^{(n+m)}\right)$ consist from objects

$$
O b \mathcal{C}\left(V^{(n+m)}\right)=\left\{V^{(n+m)}, V^{(n+m)}<i_{1}>, V^{(n+m)}<i_{2}>, \ldots\right\}
$$

being la-spacetimess, for simplicity in this work, having common $\mathrm{N}$-connection structures, and morphisms $\operatorname{MorC}\left(V^{(n+m)}\right)=\left\{n c\left(V^{(n+m)}<i_{1}>, V^{(n+m)}<i_{2}>\right)\right\}$ being chains of na-maps interrelating la-spacetimes. We point out that we can consider 
equivalent models of physical theories on every object of $\mathcal{C}\left(V^{(n+m)}\right)$. One of the main purposes of this section is to develop a d-tensor and $\mathrm{d}$-variational formalism on $\mathcal{C}\left(V^{(n+m)}\right)$, i.e. on la-multispaces, interrelated with nc-maps. Taking into account the distinguished character of geometrical objects on la-spacetimes we call tensors on $\mathcal{C}\left(V^{(n+m)}\right)$ as distinguished tensors on la-spacetime Category, or dc-tensors.

Finally, we emphasize that the presented in this Section definitions and theorems can be generalized for (super) vector bundles with arbitrary given structures of nonlinear connection, linear d-connection and metric structures [40, 43].

\section{The Nearly Autoparallel Tensor-Integral}

The aim of this Section is to define the tensor integration not only for bitensors, objects defined on the same curved space, but for dc-tensors, defined on two spaces, $V^{(n+m)}$ and $\underline{V}^{(n+m)}$, even it is necessary on la-multispaces. A. Moór tensor-integral formalism [30] having a lot of applications in classical and quantum gravity [33, 46, 17] was extended for locally isotropic multispaces in [44, 42. The unispacial locally anisotropic version is given in 40, 34, 35, 18.

Let $T_{u} V^{(n+m)}$ and $T_{\underline{u}} \underline{V}^{(n+m)}$ be tangent spaces in corresponding points $u \in$ $U \subset V^{(n+m)}$ and $\underline{u} \in \underline{U} \subset \underline{V}^{(\bar{n}+m)}$ and, respectively, $T_{u}^{*} V^{(n+m)}$ and $T_{\underline{u}}^{*} \underline{V}^{(n+m)}$ be their duals (in general, in this Section we shall not consider that a common coordinatization is introduced for open regions $U$ and $\underline{U}$ ). We call as the dc-tensors on the pair of spaces $\left(V^{(n+m)}, \underline{V}^{(n+m)}\right)$ the elements of distinguished tensor algebra

$$
\left(\otimes_{\alpha} T_{u} V^{(n+m)}\right) \otimes\left(\otimes_{\beta} T_{u}^{*} V^{(n+m)}\right) \otimes\left(\otimes_{\gamma} T_{\underline{u}} \underline{V}^{(n+m)}\right) \otimes\left(\otimes_{\delta} T_{\underline{u}}^{*} \underline{V}^{(n+m)}\right)
$$

defined over the space $V^{(n+m)} \otimes \underline{V}^{(n+m)}$, for a given $n c: V^{(n+m)} \rightarrow \underline{V}^{(n+m)}$.

We admit the convention that underlined and non-underlined indices refer, respectively, to the points $\underline{u}$ and $u$. Thus $Q_{. \underline{\alpha}}^{\beta}$, for instance, are the components of dc-tensor $Q \in T_{u} V^{(n+m)} \otimes T_{\underline{u}} \underline{V}^{(n+m)}$.

Now, we define the transport dc-tensors. Let open regions $U$ and $\underline{U}$ be homeomorphic to sphere $\mathcal{R}^{2(n+m)}$ and introduce isomorphism $\mu_{u, \underline{u}}$ between $T_{u} V^{(n+m)}$ and $T_{\underline{u}} \underline{V}^{(n+m)}$ (given by map $n c: U \rightarrow \underline{U}$ ). We consider that for every d-vector $v^{\alpha} \in T_{u} \bar{V}^{(n+m)}$ corresponds the vector $\mu_{u, \underline{u}}\left(v^{\alpha}\right)=v^{\underline{\alpha}} \in T_{\underline{u}} \underline{V}^{(n+m)}$, with components $v^{\underline{\alpha}}$ being linear functions of $v^{\alpha}$ :

$$
v^{\underline{\alpha}}=h \underline{\alpha}(u, \underline{u}) v^{\alpha}, \quad v_{\underline{\alpha}}=h_{\underline{\alpha}}^{\alpha}(\underline{u}, u) v_{\alpha},
$$

where $h_{\underline{\alpha}}^{\alpha}(\underline{u}, u)$ are the components of dc-tensor associated with $\mu_{u, \underline{u}}^{-1}$. In a similar manner we have

$$
v^{\alpha}=h_{\underline{\alpha}}^{\alpha}(\underline{u}, u) v^{\underline{\alpha}}, \quad v_{\alpha}=h \underline{\alpha}(u, \underline{u}) v_{\underline{\alpha}} .
$$

In order to reconcile just presented definitions and to assure the identity for trivial maps $V^{(n+m)} \rightarrow \underline{V}^{(n+m)}, u=\underline{u}$, the transport dc-tensors must satisfy conditions:

$$
h_{\alpha}^{\alpha}(u, \underline{u}) h_{\underline{\alpha}}^{\beta}(\underline{u}, u)=\delta_{\alpha}^{\beta}, h_{\alpha}^{\underline{\alpha}}(u, \underline{u}) h_{\underline{\beta}}^{\alpha}(\underline{u}, u)=\delta_{\underline{\beta}} \underline{\alpha}
$$


and $\lim _{\underline{\underline{u} \rightarrow u)}} h_{\underline{\alpha}}(u, \underline{u})=\delta_{\underline{\alpha}}^{\underline{\alpha}}, \quad \lim _{(\underline{u} \rightarrow u)} h_{\underline{\alpha}}^{\alpha}(\underline{u}, u)=\delta_{\underline{\alpha}}^{\alpha}$.

Let $\bar{S}_{p} \subset \bar{U} \subset \bar{V}^{(n+m)}$ is a homeomorphic to $p$-dimensional sphere and suggest that chains of na-maps are used to connect regions:

$$
U \stackrel{n c_{(1)}}{\longrightarrow} \bar{S}_{p} \stackrel{n c_{(2)}}{\longrightarrow} \underline{U} .
$$

Definition 5 The tensor integral in $\bar{u} \in \bar{S}_{p}$ of a dc-tensor $\left.N_{\varphi \cdot \bar{c}_{-} \cdot \bar{\alpha}_{1} \cdots \bar{\alpha}_{p}}^{\cdot \cdot \cdot \bar{u}}, u\right)$, completely antisymmetric on the indices $\bar{\alpha}_{1}, \ldots, \bar{\alpha}_{p}$, over domain $\bar{S}_{p}$, is defined as

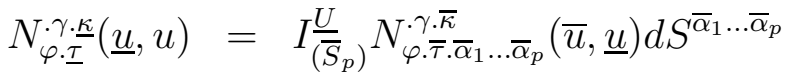

$$
\begin{aligned}
& =\int_{\left(\bar{S}_{p}\right)} h_{\underline{\tau}}^{\bar{\tau}}(\underline{u}, \bar{u}) h \frac{\kappa}{\bar{\kappa}}(\bar{u}, \underline{u}) N_{\varphi \cdot \bar{\tau} \cdot \bar{\alpha}_{1} \cdots \bar{\alpha}_{p}}^{. \cdot \bar{u}}(\bar{u}, u) d \bar{S}^{\bar{\alpha}_{1} \cdots \bar{\alpha}_{p}},
\end{aligned}
$$

where $d S^{\bar{\alpha}_{1} \cdots \bar{\alpha}_{p}}=\delta u^{\bar{\alpha}_{1}} \wedge \cdots \wedge \delta u_{p}^{\bar{\alpha}}$.

Let suppose that transport dc-tensors $h_{\alpha}^{\alpha}$ and $h_{\alpha}^{\alpha}$ admit covariant derivations of order two and postulate existence of deformation $\overline{\mathrm{d} c}$-tensor $B_{\alpha \beta}^{\cdot . \gamma}(u, \underline{u})$ satisfying relations

$$
D_{\alpha} h \frac{\beta}{\beta}(u, \underline{u})=B_{\alpha \beta}^{\cdot . \gamma}(u, \underline{u}) h \frac{\beta}{\gamma}(u, \underline{u})
$$

and, taking into account that $D_{\alpha} \delta_{\gamma}^{\beta}=0$,

$$
D_{\alpha} h_{\underline{\beta}}^{\beta}(\underline{u}, u)=-B_{\alpha \gamma}^{. \beta}(u, \underline{u}) h_{\underline{\beta}}^{\gamma}(\underline{u}, u) .
$$

By using the formulas for torsion and, respectively, curvature of connection $\Gamma_{\beta \gamma}^{\alpha}$ we can calculate next commutators:

$$
D_{[\alpha} D_{\beta]} h \frac{\gamma}{\gamma}=-\left(R_{\gamma . \alpha \beta}^{. \lambda}+T_{. \alpha \beta}^{\tau} B_{\tau \gamma}^{. \lambda}\right) h_{\lambda}^{\frac{\gamma}{\lambda}} .
$$

On the other hand from (57) one follows that

$$
D_{[\alpha} D_{\beta]} h \frac{\gamma}{\gamma}=\left(D_{[\alpha} B_{\ddot{\beta}] \gamma}^{\ddot{\lambda}}+B_{[\alpha|\tau|}^{. \lambda} B_{\ddot{\beta}] \gamma}^{\ddot{*}}\right) h_{\lambda}^{\frac{\gamma}{\lambda}},
$$

where $|\tau|$ denotes that index $\tau$ is excluded from the action of antisymmetrization [ ]. From (58) and (59) we obtain

$$
D_{[\alpha} B_{\beta] \gamma}^{. \lambda}+B_{[\beta|\gamma|} B_{\alpha] \tau}^{. . \lambda}=\left(R_{\gamma . \alpha \beta}^{\lambda \lambda}+T_{. \alpha \beta}^{\tau} B_{\tau \gamma}^{. . \lambda}\right) .
$$

Let $\bar{S}_{p}$ be the boundary of $\bar{S}_{p-1}$. The Stoke's type formula for the tensor-integral (56) is defined as

$$
I_{\bar{S}_{p}} N_{\varphi \cdot \bar{\tau} \cdot \bar{\alpha}_{1} \ldots \bar{\alpha}_{p}}^{\gamma \cdot \bar{\gamma}} d S^{\bar{\alpha}_{1} \ldots \bar{\alpha}_{p}}=I_{\bar{S}_{p+1}}{ }^{\star(p)} \bar{D}_{[\bar{\gamma} \mid} N_{\left.\varphi \cdot \bar{\tau} \cdot \mid \bar{\alpha}_{1} \ldots \bar{\alpha}_{p}\right]}^{\gamma \cdot \overline{\alpha_{1}}} d S^{\overline{\gamma \alpha_{1} \ldots \bar{\alpha}_{p}}},
$$

where

$$
\begin{aligned}
& { }^{(p)} D_{[\bar{\gamma} \mid} N_{\left.\varphi \cdot \bar{\tau} \cdot \mid \bar{\alpha}_{1} \ldots \bar{\alpha}_{p}\right]}^{\gamma \cdot \bar{\kappa}}=D_{[\bar{\gamma} \mid} N_{\left.\varphi \cdot \bar{\tau} \cdot \mid \bar{\alpha}_{1} \ldots \bar{\alpha}_{p}\right]}^{. \cdot \bar{k}}
\end{aligned}
$$

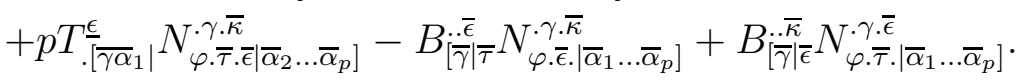


We define the dual element of the hypersurfaces element $d S^{j_{1} \ldots j_{p}}$ as

$$
d \mathcal{S}_{\beta_{1} \ldots \beta_{q-p}}=\frac{1}{p !} \epsilon_{\beta_{1} \ldots \beta_{k-p} \alpha_{1} \ldots \alpha_{p}} d S^{\alpha_{1} \ldots \alpha_{p}}
$$

where $\epsilon_{\gamma_{1} \ldots \gamma_{q}}$ is completely antisymmetric on its indices and

$$
\epsilon_{12 \ldots(n+m)}=\sqrt{|g|}, g=\operatorname{det} \mid g_{\alpha \beta \mid},
$$

$g_{\alpha \beta}$ is taken as the $\mathrm{d}$-metric (10). The dual of dc-tensor $N_{\varphi \cdot \bar{\tau} \cdot \bar{\alpha}_{1} \ldots \bar{\alpha}_{p}}^{\cdot \gamma \bar{\kappa}}$ is defined as the

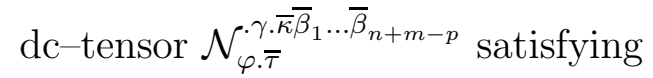

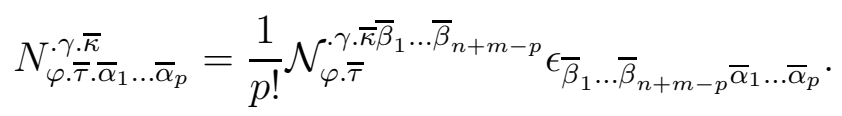

Using (61) and (62) we can write

$$
I_{\bar{S}_{p}} N_{\varphi \cdot \bar{\tau} \cdot \bar{\alpha}_{1} \ldots \bar{\alpha}_{p}}^{\cdot \cdot \bar{\kappa}} d S^{\bar{\alpha}_{1} \ldots \bar{\alpha}_{p}}=\int_{\bar{S}_{p+1}} \bar{p} D_{\bar{\gamma}} \mathcal{N}_{\varphi \cdot \bar{\tau}}^{\cdot \gamma \cdot \bar{\kappa} \bar{\beta}_{1} \ldots \bar{\beta}_{n+m-p-1} \bar{\gamma}} d \mathcal{S}_{\bar{\beta}_{1} \ldots \bar{\beta}_{n+m-p-1}},
$$

where

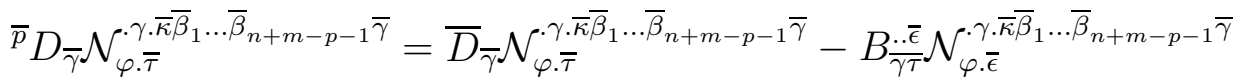

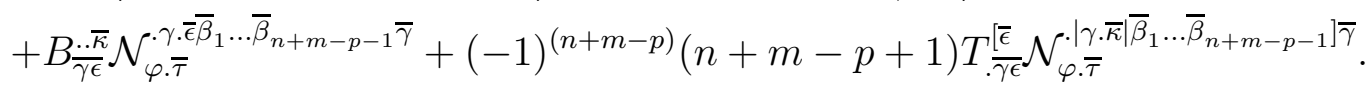

To verify the equivalence of (62) and (63) we must take in consideration that

$$
D_{\gamma} \epsilon_{\alpha_{1} \ldots \alpha_{k}}=0 \text { and } \epsilon_{\beta_{1} \ldots \beta_{n+m-p} \alpha_{1} \ldots \alpha_{p}} \epsilon^{\beta_{1} \ldots \beta_{n+m-p} \gamma_{1} \ldots \gamma_{p}}=p !(n+m-p) ! \delta_{\alpha_{1}}^{\left[\gamma_{1}\right.} \cdots \delta_{\alpha_{p}}^{\left.\gamma_{p}\right]} .
$$

The developed tensor integration formalism will be used in the next section for definition of conservation laws on spaces with local anisotropy.

\section{Tensor Integrals and Conservation Laws}

The definition of conservation laws on curved and/or locally anisotropic spaces is a challenging task because of absence of global and local groups of automorphisms of such spaces. Our main idea is to use chains of na-maps from a given, called hereafter as the fundamental, la-spacetime to an auxiliary one with trivial curvatures and torsions admitting a global group of automorphisms. The aim of this section is to formulate conservation laws for la-gravitational fields by using dc-objects and tensor-integral values, na-maps and variational calculus on the Category of laspacetimes. R. Miron and M. Anastasiei [27, 28] calculated the divergence of the energy-momentum d-tensor on vector bundles provided with $\mathrm{N}$-connection structure (the same formulas hold for (pseudo) Riemannian la-spacetimes)

$$
D_{\alpha} E_{\beta}^{\alpha}=\frac{1}{\kappa_{1}} U_{\alpha}
$$


where

$$
E_{\beta}^{\alpha}=R_{\beta}^{\alpha}-\frac{1}{2} \delta_{\beta}^{\alpha} \overleftarrow{R}
$$

is the Einstein $\mathrm{d}$-tensor, and concluded that the $\mathrm{d}$-vector

$$
U_{\alpha}=\frac{1}{2}\left(G^{\beta \delta} R_{\delta}^{\gamma}{ }_{\phi \beta} T_{\cdot \alpha \gamma}^{\phi}-G^{\beta \delta} R_{\delta}{ }^{\gamma}{ }_{\phi \alpha} T_{\cdot \beta \gamma}^{\phi}+R_{\phi}^{\beta} T_{\cdot \beta \alpha}^{\phi}\right)
$$

vanishes if and only if the $\mathrm{d}-$-connection $D$ is without torsion. On $V^{(n+m)}$ the $\mathrm{d}-$ torsion $T_{\cdot \alpha \gamma}^{\phi}$ could be effectively induced with respect to an anholonomic frame and became trivial after transition to a holonomic frame.

No wonder that conservation laws, in usual physical theories being a consequence of global (for usual gravity of local) automorphisms of the fundamental spacetime, are more sophisticate on the spaces with local anisotropy. Here it is important to emphasize the multiconnection character of la-spacetimes. For example, for a dmetric (10) on $V^{(n+m)}$ we can equivalently define an auxiliar linear connection $\tilde{D}$ constructed from by using the usual formulas for Christoffel symobls with the operators of partial differential equations (5) chainged respectively into the la-adapted ones (1). We conclude that by using auxiliary symmetric d-connections, we can also use the symmetric $\mathrm{d}$-connection $\gamma_{\beta \gamma}^{\alpha}$ from (22) we construct a model of lagravity which looks like locally isotropic on the spacetime $V^{(n+m)}$. More general gravitational models with local anisotropy can be obtained by using deformations of connection $\tilde{\Gamma}_{\cdot \beta \gamma}^{\alpha}$,

$$
\Gamma_{\beta \gamma}^{\alpha}=\tilde{\Gamma}_{\cdot \beta \gamma}^{\alpha}+P_{\beta \gamma}^{\alpha}+Q_{\beta \gamma}^{\alpha},
$$

were, for simplicity, $\Gamma_{\beta \gamma}^{\alpha}$ is chosen to be also metric and satisfy the Einstein equations (15). The d-vector $U_{\alpha}$ is interpreted as an effective source of local anisotropy on $V^{(n+m)}$ satisfying the generalized conservation laws (64). The deformation $\mathrm{d}-$ tensor $P_{\beta \gamma}^{\alpha}$ is could be generated (or not) by deformations of type (29)-(33) for na-maps.

¿From (56) we obtain a tensor integral on $\mathcal{C}\left(V^{(n+m)}\right)$ of a d-tensor:

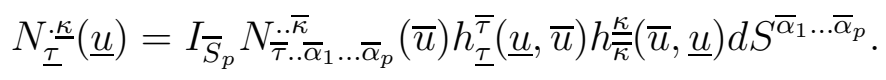

We point out that tensor-integrals can be defined not only for dc-tensors but and for d-tensors on $V^{(n+m)}$. Really, suppressing indices $\varphi$ and $\gamma$ in (62) and (63), considering instead of a deformation dc-tensor a deformation tensor

$$
B_{\alpha \beta}^{. \cdot \gamma}(u, \underline{u})=B_{\alpha \beta}^{. \cdot \gamma}(u)=P_{. \alpha \beta}^{\gamma}(u)
$$

(we consider deformations induced by a nc-transform) and integration $I_{S_{p}} \ldots d S^{\alpha_{1} \ldots \alpha_{p}}$ in la-spacetime $V^{(n+m)}$ we obtain from (56) a tensor-integral on $\mathcal{C}\left(V^{(n+m)}\right)$ of a d-tensor:

$$
N_{\underline{\underline{\tau}}}^{\underline{\kappa}}(\underline{u})=I_{S_{p}} N_{\tau \cdot \alpha_{1} \ldots \alpha_{p}}^{. \kappa}(u) h_{\underline{\tau}}^{\tau}(\underline{u}, u) h \underline{\kappa}(u, \underline{u}) d S^{\alpha_{1} \ldots \alpha_{p}} .
$$

Taking into account (59) we can calculate that curvature

$$
\underline{R}_{\gamma . \alpha \beta}^{. \lambda}=D_{[\beta} B_{\alpha] \gamma}^{. . \lambda}+B_{[\alpha|\gamma|}^{. \tau} B_{\beta] \tau}^{. . \lambda}+T_{. \alpha \beta}^{\tau . . .} B_{\tau \gamma}^{. . \lambda}
$$


of connection $\underline{\Gamma}_{. \alpha \beta}^{\gamma}(u)=\Gamma_{. \alpha \beta}^{\gamma}(u)+B_{\alpha \beta}^{. . \gamma}(u)$, with $B_{\alpha \beta}^{. \cdot \gamma}(u)$ taken from (65), vanishes, $\underline{R}_{\gamma . \alpha \beta}^{\lambda}=0$. So, we can conclude that a la-spacetime $V^{(n+m)}$ admits a tensor integral structure on $\mathcal{C}\left(V^{(n+m)}\right)$ for d-tensors associated to the deformation tensor $B_{\alpha \beta}^{. . \gamma}(u)$ if the nc-image $\underline{V}^{(n+m)}$ is locally parallelizable. That way we generalize the one space tensor integral constructions from [17, 18, 35, were the possibility to introduce tensor integral structure on a curved space was restricted by the condition that this space is locally parallelizable. For $q=n+m$ the relations (63), written for $\mathrm{d}-$ tensor $\mathcal{N}_{\underline{\alpha}}^{\underline{\beta} \underline{\gamma}}$ (we change indices $\bar{\alpha}, \bar{\beta}, \ldots$ into $\underline{\alpha}, \underline{\beta}, \ldots$ ) extend the Gauss formula on $\mathcal{C}\left(V^{(n+m)}\right)$ :

$$
I_{S_{q-1}} \mathcal{N}_{\underline{\alpha}}^{\cdot \underline{\beta \gamma}} d \mathcal{S}_{\underline{\gamma}}=I_{\underline{S}} \frac{q-1}{\underline{\underline{\tau}}} D_{\underline{\underline{\tau}}} \mathcal{N}_{\underline{\underline{\alpha}}}^{\underline{\beta} \underline{\tau}} d \underline{V},
$$

where $d \underline{V}=\sqrt{\left|\underline{g} \underline{g}_{\alpha \beta}\right|} d \underline{u}^{1} \ldots d \underline{u}^{q}$ and

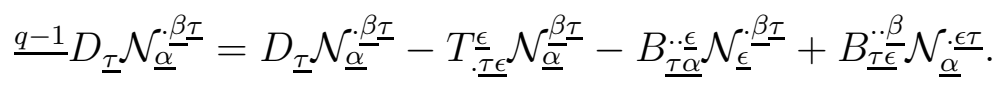

Let consider physical values $N_{\underline{\alpha}}^{\cdot \frac{\beta}{}}$ on $\underline{V}^{(n+m)}$ defined on its density $\mathcal{N}_{\underline{\alpha}}^{\cdot \frac{\beta \gamma}{\gamma}}$, i. e.

$$
N_{\underline{\alpha}}^{\underline{\beta}}=I_{\underline{S}_{q-1}} \mathcal{N}_{\underline{\alpha}}^{\cdot \frac{\beta \gamma}{}} d \mathcal{S}_{\underline{\gamma}}
$$

with this conservation law (due to (66)):

$$
\underline{q-1} D_{\underline{\gamma}} \mathcal{N}_{\underline{\underline{\alpha}}}^{\underline{\beta \gamma}}=0 .
$$

We note that these conservation laws differ from covariant conservation laws for well known physical values such as density of electric current or of energy- momentum tensor. For example, taking the density $E_{\beta}^{\cdot \gamma}$, with corresponding to (67) and (69) conservation law,

$$
{ }^{q-1} D_{\underline{\gamma}} E_{\underline{\beta}}^{\frac{\gamma}{\beta}}=D_{\underline{\gamma}} E_{\underline{\beta}}^{\frac{\gamma}{\beta}}-T_{. \underline{\tau}}^{\tau} E_{\underline{\underline{\beta}}}^{\cdot \epsilon}-B_{\underline{\tau}}^{\cdot \cdot \underline{\epsilon}} E_{\underline{\epsilon}}^{\tau}=0,
$$

we can define values (see (66) and (68))

$$
\mathcal{P}_{\alpha}=I_{\underline{S}_{q-1}} E_{\underline{\alpha}}^{\cdot \underline{\gamma}} d \mathcal{S}_{\underline{\gamma}}
$$

The defined conservation laws (70) for $E_{\underline{\underline{\beta}}}^{\cdot \underline{\epsilon}}$ are not related with those for energymomentum tensor $E_{\alpha}^{\cdot \gamma}$ from the Einstein equations for the almost Hermitian gravity [27, 28] or with a $\tilde{E}_{\alpha \beta}^{\alpha}$ with vanishing divergence $D_{\gamma} \tilde{E}_{\alpha}^{\cdot \gamma}=0$. So $\tilde{E}_{\alpha}^{\cdot \gamma} \neq E_{\alpha}^{\cdot \gamma}$. A similar conclusion was made in [17] for the unispacial locally isotropic tensor integral. In the case of multispatial tensor integration we have another possibility (firstly pointed in [44, 35] for Einstein-Cartan spaces), namely, to identify $E_{\underline{\beta}}^{-\gamma}$ from (70) with the na-image of $E_{\beta}^{\cdot \gamma}$ on la-spacetime $V^{(n+m)}$. We shall consider this construction in the next Section. 


\section{Conservation Laws for $\mathrm{Na}-$ Backrounds}

Let us consider a fixed background la-spacetime $\underline{V}^{(n+m)}$ with given metric $\underline{g}_{\alpha \beta}=$ $\left(\underline{g}_{i j}, \underline{h}_{a b}\right)$ and d-connection $\underline{\tilde{\Gamma}}_{\cdot \beta \gamma}^{\alpha}$. For simplicity, we suppose that the metricity conditions are satisfied and that the connection is torsionless and with vanishing curvature. Considering a nc-transform from the fundamental la-space $V^{(n+m)}$ to an auxiliary one $\underline{V}^{(n+m)}$ we are interested in the equivalents of the Einstein equations on $\underline{V}^{(n+m)}$.

We suppose that a part of gravitational degrees of freedom is "pumped out" into the dynamics of deformation d-tensors for $\mathrm{d}$-connection, $P_{\beta \gamma}^{\alpha}$, and metric, $B^{\alpha \beta}=\left(b^{i j}, b^{a b}\right)$. The remained part of degrees of freedom is coded into the metric $\underline{g}_{\alpha \beta}$ and d-connection $\underline{\tilde{\Gamma}}_{\cdot \beta \gamma}^{\alpha}$.

Following [19, 34] we apply the first order formalism and consider $B^{\alpha \beta}$ and $P^{\alpha}{ }_{\beta \gamma}$ as independent variables on $\underline{V}^{(n+m)}$. Using notations

$$
\begin{gathered}
P_{\alpha}=P_{\beta \alpha}^{\beta}, \quad \Gamma_{\alpha}=\Gamma_{\beta \alpha}^{\beta}, \\
\hat{B}^{\alpha \beta}=\sqrt{|g|} B^{\alpha \beta}, \widehat{g}^{\alpha \beta}=\sqrt{|g|} g^{\alpha \beta}, \underline{g}^{\alpha \beta}=\sqrt{|\underline{g}|} \underline{g}^{\alpha \beta}
\end{gathered}
$$

and making identifications

$$
\hat{B}^{\alpha \beta}+\underline{\widehat{g}}^{\alpha \beta}=\widehat{g}^{\alpha \beta}, \quad \underline{\Gamma}_{\cdot \beta \gamma}^{\alpha}-P_{\beta \gamma}^{\alpha}=\Gamma_{\beta \gamma}^{\alpha},
$$

we take the action of la-gravitational field on $\underline{V}^{(n+m)}$ in this form:

$$
\underline{\mathcal{S}}^{(g)}=-\left(2 c \kappa_{1}\right)^{-1} \int \delta^{q} u \underline{\mathcal{L}}^{(g)},
$$

where

$$
\underline{\mathcal{L}}^{(g)}=\hat{B}^{\alpha \beta}\left(D_{\beta} P_{\alpha}-D_{\tau} P^{\tau}{ }_{\alpha \beta}\right)+\left(\underline{\hat{g}}^{\alpha \beta}+\hat{B}^{\alpha \beta}\right)\left(P_{\tau} P^{\tau}{ }_{\alpha \beta}-P_{\alpha \kappa}^{\alpha} P_{\beta \tau}^{\kappa}\right)
$$

and the interaction constant is taken $\kappa_{1}=\frac{4 \pi}{c^{4}} k, \quad(c$ is the light constant and $k$ is Newton constant) in order to obtain concordance with the Einstein theory in the locally isotropic limit.

We construct on $\underline{V}^{(n+m)}$ a la-gravitational theory with matter fields (denoted as $\varphi_{A}$ with $A$ being a general index) interactions by postulating this Lagrangian density for matter fields

$$
\underline{\mathcal{L}}^{(m)}=\underline{\mathcal{L}}^{(m)}\left[\underline{\widehat{g}}^{\alpha \beta}+\hat{B}^{\alpha \beta} ; \frac{\delta}{\delta u^{\gamma}}\left(\underline{\widehat{g}}^{\alpha \beta}+\hat{B}^{\alpha \beta}\right) ; \varphi_{A} ; \frac{\delta \varphi_{A}}{\delta u^{\tau}}\right] .
$$

Starting from (71) and (72) the total action of la-gravity on $\underline{V}^{(n+m)}$ is written as

$$
\underline{\mathcal{S}}=\left(2 c \kappa_{1}\right)^{-1} \int \delta^{q} u \underline{\mathcal{L}}^{(g)}+c^{-1} \int \delta^{(m)} \underline{\mathcal{L}}^{(m)} .
$$

Applying variational procedure on $\underline{V}^{(n+m)}$, similar to that presented in 19 but in our case adapted to $\mathrm{N}$-connection by using derivations (5) instead of partial derivations (11), we derive from (73) the la-gravitational field equations

$$
\boldsymbol{\Theta}_{\alpha \beta}=\kappa_{1}\left(\underline{\mathbf{t}}_{\alpha \beta}+\underline{\mathbf{T}}_{\alpha \beta}\right)
$$


and matter field equations

$$
\frac{\triangle \underline{\mathcal{L}}^{(m)}}{\triangle \varphi_{A}}=0
$$

where $\triangle / \triangle \varphi_{A}$ denotes the variational derivation.

In (74) we have introduced these values: the energy-momentum $d$-tensor for la-gravitational field

$$
\begin{gathered}
\kappa_{1} \underline{\mathbf{t}}_{\alpha \beta}=(\sqrt{|g|})^{-1} \frac{\triangle \underline{\mathcal{L}}^{(g)}}{\triangle g^{\alpha \beta}}=K_{\alpha \beta}+P^{\gamma}{ }_{\alpha \beta} P_{\gamma}-P^{\gamma}{ }_{\alpha \tau} P^{\tau}{ }_{\beta \gamma}+ \\
\frac{1}{2} \underline{g}_{\alpha \beta} \underline{g}^{\gamma \tau}\left(P^{\phi}{ }_{\gamma \tau} P_{\phi}-P^{\phi}{ }_{\gamma \epsilon} P_{\phi \tau}^{\epsilon}\right)
\end{gathered}
$$

(where

$$
\begin{gathered}
K_{\alpha \beta}=\underline{D}_{\gamma} K_{\alpha \beta}^{\gamma}, \\
2 K_{\alpha \beta}^{\gamma}=-B^{\tau \gamma} P^{\epsilon}{ }_{\tau(\alpha} \underline{g}_{\beta) \epsilon}-B^{\tau \epsilon} P^{\gamma}{ }_{\epsilon(\alpha} \underline{g}_{\beta) \tau}+ \\
\left.\underline{g}^{\gamma \epsilon} h_{\epsilon(\alpha} P_{\beta)}+\underline{g}^{\gamma \tau} \underline{g}^{\epsilon \phi} P^{\varphi}{ }_{\phi \tau} \underline{G}_{\varphi(\alpha} B_{\beta) \epsilon}+\underline{g}_{\alpha \beta} B^{\tau \epsilon} P^{\gamma}{ }_{\tau \epsilon}-B_{\alpha \beta} P^{\gamma}\right), \\
2 \Theta=\underline{D}^{\tau} \underline{D}_{\tau} B_{\alpha \beta}+\underline{g}_{\alpha \beta} \underline{D}^{\tau} \underline{D}^{\epsilon} B_{\tau \epsilon}-\underline{g}^{\tau \epsilon} \underline{D}_{\epsilon} \underline{D}_{(\alpha} B_{\beta) \tau}
\end{gathered}
$$

and the energy-momentum $\mathrm{d}$-tensor of matter

$$
\underline{\mathbf{T}}_{\alpha \beta}=2 \frac{\triangle \mathcal{L}^{(m)}}{\triangle \underline{\hat{g}}^{\alpha \beta}}-\underline{g}_{\alpha \beta} \underline{g}^{\gamma \delta} \frac{\triangle \mathcal{L}^{(m)}}{\triangle \underline{\hat{g}}^{\gamma \delta}} .
$$

As a consequence of $(\sqrt{75})-(77)$ we obtain the d-covariant on $\underline{V}^{(n+m)}$ conservation laws

$$
\underline{D}_{\alpha}\left(\underline{\mathbf{t}}^{\alpha \beta}+\underline{\mathbf{T}}^{\alpha \beta}\right)=0 .
$$

We have postulated the Lagrangian density of matter fields (72) in a form as to treat $\underline{\mathbf{t}}^{\alpha \beta}+\underline{\mathbf{T}}^{\alpha \beta}$ as the source in ([74).

Now we formulate the main results of this Section:

Proposition 1 The dynamics of the la-gravitational fields, modeled as solutions of the Einstein equations (15) and of matter field equations on la-spacetime $V^{(n+m)}$, can be locally equivalently modeled on a background la-spacetime $\underline{V}^{(n+m)}$ provided with a trivial d-connection and metric structure (with vanishing d-tensors of torsion and curvature) by equations (74) and (75) on condition that the deformation tensor $P^{\alpha}{ }_{\beta \gamma}$ is a solution of the Cauchy problem posed for the basic equations for a chain of na-maps from $V^{(n+m)}$ to $\underline{V}^{(n+m)}$.

Proposition 2 The local d-tensor conservation laws for Einstein la-gravitational fields can be written in the form (78) for both la-gravitational (76) and matter (77) energy-momentum $d$-tensors. These laws are $d$-covariant on the background space $\underline{V}^{(n+m)}$ and must be completed with invariant conditions of type (34)-((37)) for every deformation parameters of a chain of na-maps from $V^{(n+m)}$ to $\underline{V}^{(n+m)}$. 
The above presented considerations consist proofs of both propositions.

We emphasize that the nonlocalization of both locally anisotropic and isotropic gravitational energy-momentum values on the fundamental (locally anisotropic or isotropic) spacetime $V^{(n+m)}$ is a consequence of the absence of global group automorphisms for generic curved spaces. Considering gravitational theories from the view of multispaces and their mutual maps (directed by the basic geometric structures on $V^{(n+m)}$ such as $\mathrm{N}$-connection, d-connection, d-torsion and d-curvature components, see the coefficients for basic na-equations (29)-(33)), we can formulate local d-tensor conservation laws on auxiliary globally automorphic spaces being related with some covering regions of the spacetime $V^{(n+m)}$ by means of chains of na-maps. Finally, we remark that as a matter of principle we can also use $\mathrm{d}$-connection deformations in order to modelate the la-gravitational interactions with nonvanishing torsion and nonmetricity. In this case we must introduce a corresponding source in (78) and define generalized conservation laws as in (64).

\section{Einstein Spaces Generated by Finsler Like Met- rics}

In this Section we analyze the conditions when four dimensional (4D) vacuum and non-vacuum solutions of the Einstein equations can be induced by Finsler like metrics depending on three variables; we construct such solutions in explicit form.

\subsection{Two dimensional Finsler metrics}

There is a class of 2D Finsler metrics

$$
h_{a b}\left(x^{i}, y^{c}\right)=\frac{1}{2} \frac{\partial^{2} F^{2}\left(x^{i}, y^{c}\right)}{\partial y^{a} \partial y^{b}}
$$

generated by the so-called Finsler metric function $F=F\left(x^{i}, y^{c}\right)$, where the indices $i, j, k, \ldots$ run values 1 and 2 on a $2 \mathrm{D}$ base manifold $V^{(2)}$ and $y$-coordinate indices $a, b, c, \ldots=3,4$ are used for $2 \mathrm{D}$ fibers $Y_{x}$ of the tangent bundle $T V^{(2)}$.. Because for Finsler spaces the function $F$ is homogeneous on $y$-variables we can express

$$
F\left(x^{1}, x^{2}, y^{3}, y^{4}\right)=y^{3} f\left(x^{1}, x^{2}, z\right),
$$

where $z=y^{4} / y^{3}$ and

$$
f=f(x, z)=f\left(x^{1}, x^{2}, z\right) \doteq F\left(x^{1}, x^{2}, 1, z\right)
$$

By introducing the function $K(x, z)=\left(f f^{\prime}\right)^{\prime}$, where the 'prime' denotes the partial derivation on $z$, the metric coefficients (79) are computed

$$
h_{3}=h_{33}=K z^{2}-2 f f^{\prime} z+f^{2}, h=h_{34}=-K z+f f^{\prime}, h_{4}=h_{44}=K .
$$


We note that if the 2D Finsler metric coefficients formally depended on four variables, by introducing the function $f\left(x^{1}, x^{2}, z\right)$ one has obtained an explicit dependence only on three coordinates.

Consider a vertical 2D d-metric

$$
h_{a b}\left(x^{i}, z\right)=\left(\begin{array}{cc}
h_{3}\left(x^{i}, z\right) & h\left(x^{i}, z\right) \\
h\left(x^{i}, z\right) & h_{4}\left(x^{i}, z\right)
\end{array}\right)
$$

which, by applying a matrix transform (21) can be diagonalized

$$
h_{a^{\prime} b^{\prime}}\left(x^{i}, z\right)=\left(\begin{array}{cc}
\lambda_{3}\left(x^{i}, z\right) & 0 \\
0 & \lambda_{4}\left(x^{i}, z\right)
\end{array}\right) .
$$

We can generate by a Finsler metric function a 2D diagonal (pseudo) Riemannian metric

$$
h_{a^{\prime} b^{\prime}}(t, r)=\left(\begin{array}{cc}
\lambda_{3}\left(x^{i}\right) & 0 \\
0 & \lambda_{4}\left(x^{i}\right)
\end{array}\right)
$$

depending only on coordinates $x=\left\{x^{i}\right\}$ if we choose the square of $f$-function $s\left(x^{i}, z\right)=f^{2}\left(x^{i}, z\right)$ from (80) to be (see formulas (81))

$$
s\left(x^{i}, z\right)=z^{2} \lambda_{2}\left(x^{i}\right)+\lambda_{1}\left(x^{i}\right) .
$$

This is the simplest case when a $2 \mathrm{D}$ diagonal metric (84) is defined by a trivial Finsler squared $f$-function (85) depending on $z^{2}$ and two functions $\lambda_{1,2}\left(x^{i}\right)$.

The problem of definition of a corresponding Finsler metric function becames more difficult if we try to generate not a diagonal 2D metric (84) depending only on two variables $\left(x^{i}\right)$, but a nondiagonal one depending on three variables $\left(x^{i}, z\right)$ (see (82)). There are three classes of such type Finsler generated 2D metrics.

\subsubsection{Euler nonhomogeneous equations and Finsler metrics}

The first class of 2D Finsler metric is defined by the condition when the function $s\left(x^{i}, z\right)$ is chosen as to solve the first equation in (81) when the coefficient $a_{1}\left(x^{i}, z\right)$ of a nondiagonal 2D d-metric (82) are prescribed. The rest of components of the vertical $\mathrm{d}$-metric, $b_{1}\left(x^{i}, z\right)$ and $h\left(x^{i}, z\right)$, are not arbitrary ones but they must be found by using partial derivatives $s^{\prime}=\partial s / \partial z$ and $s^{\prime \prime}=\partial^{2} s / \partial z^{2}$, in correspondence with the formulas (81).

The basic equation is

$$
z^{2} s^{\prime \prime}-2 z s^{\prime}+2 s=2 a_{1}
$$

which for $a_{1}=0$ and variables $x^{i}$ treated as some parameters is the so-called Euler equation [21] having solutions of type

$$
C_{1}\left(x^{i}\right) z^{2}+C_{2}\left(x^{i}\right) z .
$$

By integrating on the $z$-variable we can construct the solution $s_{(a 1)}$ of (86) for a nonvanishing right part,

$$
s_{(a 1)}\left(x^{i}, z\right)=z C_{(0)}\left(x^{i}\right)+z^{2} C_{(1)}\left(x^{i}\right)+z \cdot \int_{\text {const } 2}^{z} d \zeta \int_{\text {const } 1}^{\zeta} d \tau \frac{a_{1}\left(x^{i}, \tau\right)}{\tau^{3}}
$$


where $C_{(0)}\left(x^{i}\right)$ and $C_{(1)}\left(x^{i}\right)$ are some arbitrary functions, the index $(a 1)$ emphasizes that the Finsler metric is associated to the value $a_{1}\left(x^{i}, \tau\right)$ and the const 1 and const 2 in the integrals should be chosen from some boundary conditions.

The 2D d-metric coefficients $h\left(x^{i}, \tau\right)$ and $b_{1}\left(x^{i}, \tau\right)$ are computed

$$
b_{1}=\frac{1}{2} s^{\prime \prime} \text { and } h=-z b_{1}+\frac{s^{\prime}}{2}
$$

where $s=s_{\left(a_{1}\right)}$.

\subsubsection{The simplest case}

If the coefficient $b_{1}\left(x^{i}, \tau\right)$ is given, the squared 2D metric Finsler function is to be found from the last formula in (81), $b_{1}=s^{\prime \prime} / 2$. By considering the coordinates $(r, t)$ as parameters, and integrating on $z$ we obtain

$$
s_{(b)}=2 \int_{\text {const } 1}^{z} d \tau \int_{\text {const } 2}^{\tau} d \vartheta b_{1}\left(x^{i}, \vartheta\right)+z S_{(0)}\left(x^{i}\right)+S_{(1)}\left(x^{i}\right),
$$

where $S_{(0)}\left(x^{i}\right)$ and $S_{(1)}\left(x^{i}\right)$ are some functions on variables $x^{i}$.

The corresponding $2 \mathrm{D}$ vertical $\mathrm{d}$-metric coefficients $h\left(x^{i}, \tau\right)$ and $a_{1}\left(x^{i}, \tau\right)$ are computed

$$
a_{1}=s-z^{2} b_{1}-2 z h \text { and } h=-z b_{1}+\frac{s^{\prime}}{2}
$$

where $s=s_{(b)}$.

\subsubsection{Prescribed nondiagonal coefficients}

In this case one choose the coefficient $h\left(x^{i}, \tau\right)$ for definition of the squared Finsler metric function $s\left(x^{i}, \tau\right)$. As the basic equation we consider the equation

$$
z s^{\prime \prime}-s^{\prime}=-h
$$

which has the solution

$$
s_{(h)}=\varphi_{1}\left(x^{i}\right)+z^{2} \varphi_{2}\left(x^{i}\right)-2 \int_{\text {const } 1}^{z} \zeta d \zeta \cdot \int_{\text {const } 2}^{\zeta} d \tau \frac{h\left(x^{i}, \tau\right)}{\tau^{2}}
$$

depending on two arbitrary functions $\varphi_{1,2}(t, r)$.

The explicit formulas for the rest of $2 \mathrm{D}$ vertical $\mathrm{d}$-metric coefficients $b_{1}\left(x^{i}, \tau\right)$ and $a_{1}\left(x^{i}, \tau\right)$ follows from

$$
h=-z b_{1}+\frac{s^{\prime}}{2} \text { and } a_{1}=s-z^{2} b_{1}-2 z h
$$

where $s=s_{(h)}$. 


\subsection{An ansatz for Finsler like vacuum solutions}

Let us consider a particular case of metrics (3) are generated as generalized Lagrange metrics (20) by a diagonalization transform (21) of a Finsler induced v-metric (83)

$$
\left[\begin{array}{cccc}
g_{1}+q_{1}{ }^{2} h_{3}+n_{1}{ }^{2} \lambda_{4} & 0 & q_{1} \lambda_{3} & n_{1} \lambda_{4} \\
0 & 1+q_{2}{ }^{2} \lambda_{3}+n_{2}{ }^{2} \lambda_{4} & q_{2} \lambda_{3} & n_{2} \lambda_{4} \\
q_{1} \lambda_{3} & q_{2} \lambda_{3} & \lambda_{3} & 0 \\
n_{1} \lambda_{4} & n_{2} \lambda_{4} & 0 & \lambda_{4}
\end{array}\right]
$$

with coefficients being some functions of necessary smooth class $g_{1}=g_{1}\left(x^{2}\right), g_{2}=$ $1, q_{i}=q_{i}\left(x^{j}, z\right), n_{i}=n_{i}\left(x^{j}, z\right), \lambda_{3}=\lambda_{3}\left(x^{j}, z\right)$ and $\lambda_{4}=\lambda_{4}\left(x^{j}\right)$. Latin indices run respectively $i, j, k, \ldots=1,2$ and $a, b, c, \ldots=3,4$ and the local coordinates are denoted $u^{\alpha}=\left(x^{i}, y^{3}=z, y^{4}\right)$, where one from the coordinates $x^{1}, z$ and $y^{4}$ could be treated as a timelike coordinate. A metric (88) is diagonalized,

$$
\delta s^{2}=g_{1}\left(x^{2}\right)\left(d x^{1}\right)^{2}+\left(d x^{2}\right)^{2}+\lambda_{a}\left(x^{j}, z\right)\left(\delta y^{a}\right)^{2},
$$

with respect to corresponding anholonomic frames (5) and (5), here we write down only the 'elongated' differentials

$$
\delta z=d z+q_{i}\left(x^{j}, z\right) d x^{i}, \delta y^{4}=d y^{4}+n_{i}\left(x^{j}, z\right) d x^{i} .
$$

The system of Einstein field equations (15) reduces to four nontrivial second order partial differential equations on $z$ for functions $q_{i}\left(x^{j}, z\right), n_{i}\left(x^{j}, z\right), \lambda_{3}\left(x^{j}, z\right)$ and $\lambda_{4}\left(x^{j}\right)$

$$
\begin{aligned}
P_{3 i} & =\frac{q_{i}}{2 \lambda_{3}}\left[\frac{1}{\lambda_{3}}\left(\frac{\partial \lambda_{3}}{\partial z}\right)^{2}-\frac{\partial^{2} \lambda_{3}}{\partial z^{2}}\right], \\
P_{4 i} & =\frac{\lambda_{4}}{4 \lambda_{3}}\left[\frac{\partial n_{i}}{\partial z} \frac{\partial \lambda_{3}}{\partial z}-2 \frac{\partial^{2} n_{i}}{\partial z^{2}}\right] .
\end{aligned}
$$

There are two possibilities to satisfy the equations (90): $1 /$ If the function $\lambda_{3}\left(x^{j}, z\right)$ is a nonvanishing solution of

$$
\frac{1}{\lambda_{3}}\left(\frac{\partial \lambda_{3}}{\partial z}\right)^{2}-\frac{\partial^{2} \lambda_{3}}{\partial z^{2}}=0,
$$

the $\mathrm{N}$-connection coefficients $q_{i}\left(x^{j}, z\right)$ could take arbitrary values in correspondence to a stated Cauchy problem. 2/ The coefficients $q_{i}\left(x^{j}, z\right) \equiv 0$ if the function $\lambda_{3}$ das not satisfy the condition (92); we have only one anisotropic direction distinguished by some nontrivial functions $n_{i}\left(x^{j}, z\right)$.

The general solution of (91) is written

$$
n_{i}\left(x^{j}, z\right)=p_{i(0)}\left(x^{i}\right) \int_{0}^{z} \exp \left[\frac{\lambda_{3}\left(x^{j}, \zeta\right)}{2}\right] d \zeta+n_{i(0)}\left(x^{i}\right)
$$

where the functions $p_{i(0)}\left(x^{i}\right)$ and $n_{i(0)}\left(x^{i}\right)$ have to be defined from some boundary (initial) conditions. 
We conclude this Section by formulating the rule for generation by Finsler like metrics of vacuum solutions of the Einstein equations. Firstly, we take a Finsler metric function (80) and following (81) we induce a nondiagonal $2 \mathrm{D} \mathrm{v}-$ metric (82). Diagonalizing (21), we obtain a h-metric (83). If the induced coefficient $\lambda_{4}$ depends only on horizontal variables $x^{j}$, the ansatz (88) solves the vacuum Einsten equations under the conditions that the functions $q_{i}\left(x^{j}, z\right), n_{i}\left(x^{j}, z\right), \lambda_{3}\left(x^{j}, z\right)$ and $\lambda_{4}\left(x^{j}\right)$ satisfy the conditions (92) and (93). Instead of starting the procedure by fixing the Finsler metric function we can fix a necessary type coeffcient $h_{3}\left(h\right.$ or $\left.h_{4}\right)$ and then, as was stated in subsection 8.1.1 (8.1.2 or 8.1.3), we must define the corresponding class of Finsler metrics. Finally, diagonalizing the $\mathrm{v}$-metric, we obtain the coefficients which must be put into the ansatz (88).

We restricted our constructions only for some trivial Finsler like induced hcomponents of d-metrics, for simplicity, considering h-components of type $g_{i j}=$ $\operatorname{diag}\left[a_{1}\left(x^{2}\right), 1\right]$. To induce more general Finsler like $\mathrm{h}$-metrics is possible by a similar to the presented for $\mathrm{v}$-subspaces procedure.

\subsection{Non vacuum locally anisotropic solutions}

In this subsection we generalize the ansatz in order to induce non-vacuum solutions of the Einstein equations (88). We consider a 4D metric parametrized

$$
\left[\begin{array}{cccc}
g_{1}+q_{1}{ }^{2} h_{3}+n_{1}{ }^{2} \lambda_{4} & 0 & q_{1} \lambda_{3} & n_{1} \lambda_{4} \\
0 & g_{2}+q_{2}{ }^{2} \lambda_{3}+n_{2}{ }^{2} \lambda_{4} & q_{2} \lambda_{3} & n_{2} \lambda_{4} \\
q_{1} \lambda_{3} & q_{2} \lambda_{3} & \lambda_{3} & 0 \\
n_{1} \lambda_{4} & n_{2} \lambda_{4} & 0 & \lambda_{4}
\end{array}\right]
$$

with the coefficients being some functions of necessary smooth class $g_{1}=-\alpha(r), g_{2}=$ $1 / \alpha(r), q_{i}=q_{i}\left(x^{j}, z\right), n_{i}=n_{i}\left(x^{j}, z\right), \lambda_{3}=\lambda_{3}\left(x^{j}, z\right)$ and $\lambda_{4}=\lambda_{4}\left(x^{j}, z\right)$ where the $\mathrm{h}-$ coordinates are denoted $x^{1}=t$ (the time like coordinate) and $x^{2}=r$. Our aim is to define the function $\alpha(r)$ which gives a solution of the Einstein equations with diagonal energy momentum d-tensor

$$
\Upsilon_{\gamma}^{\beta}=\left[-\varepsilon, p_{2}, p_{3}, p_{4}\right]
$$

for a matter state when $p_{2}=-\varepsilon$ and $p_{3}=p_{4}$.

Putting these values of $h-$ metric into (13) we compute

$$
R_{1}^{1}=R_{2}^{2}=-\frac{1}{2} \cdot \alpha
$$

where the dot denote the partial derivative on $r$. Considering the $2 \mathrm{D}$ h-subspace to be of constant negative scalar curvature,

$$
\widehat{R}=2 R_{1}^{1}=-\widetilde{m}^{2},
$$

and that the Einstein la-equations (15) are satisfied we obtain the relation

$$
\cdot \alpha=\widetilde{m}^{2}=\kappa \Upsilon_{3}^{3}=\kappa \Upsilon_{4}^{4}
$$


The solution of (95) is written in the form $\alpha=\left(\widetilde{m}^{2} r^{2}-M\right)$ which defines a 2D h-metric

$$
d s_{(h)}^{2}=-\left(\widetilde{m}^{2} r^{2}-M\right) d t^{2}+\left(\widetilde{m}^{2} r^{2}-M\right)^{-1} d r^{2}
$$

being similar to a black hole solution in 2D Jackiw-Teitelboim gravity [20] and display many of attributes of black holes [24, 15, 23] with that difference that the constant $\widetilde{m}$ is defined by $4 \mathrm{D}$ physical values in $\mathrm{v}-$-subspace and for definiteness of the theory the $\mathrm{h}$-metric should be supplied with the equations for the $\mathrm{v}$-components of the $\mathrm{d}$-metric which in our case is

$$
\frac{\partial^{2} \lambda_{4}}{\partial z^{2}}-\frac{1}{2 \lambda_{4}}\left(\frac{\partial \lambda_{4}}{\partial z}\right)^{2}-\frac{1}{2 \lambda_{3}}\left(\frac{\partial \lambda_{3}}{\partial z}\right)\left(\frac{\partial \lambda_{4}}{\partial z}\right)+\frac{\kappa \varepsilon}{2} \lambda_{3} \lambda_{4}=0
$$

Prescribing one of the functions $\lambda_{3}$, or $\lambda_{4}$, the second one is to be defined by integration on the $z$-variable (see detailes in [41]). One has a forth order partial differential equation for the metric function $f\left(x^{i}, z\right)$, see (80) if we try to induce the horizontal part in a pure Finsler like manner.

\section{Nearly Conformally Flat Gravitational Fields}

We analyze chains of na-maps which by corresponding deformation parameters and deformations of connections induce a vacuum d-metric (89) (for non-vacuum metrics considerations are similar).

The nontrivial canonical d-connection coefficients (11) and d-torsions (12) are respectively computed

$$
\begin{aligned}
L_{12}^{1} & =L_{21}^{1}=\frac{1}{2} \frac{\partial \ln \left|a_{1}\right|}{\partial x^{2}}, L_{22}^{2}=-\frac{1}{2} \frac{\partial a_{1}}{\partial x^{2}} ; C_{33}^{3}=\frac{1}{2} \frac{\partial \ln \left|\lambda_{3}\right|}{\partial z} ; \\
L^{3}{ }_{3 i} & =\frac{1}{2 \lambda_{3}}\left(\frac{\partial \lambda_{3}}{\partial x^{i}}-q_{i} \frac{\partial h_{3}}{\partial z}\right), L^{3}{ }_{4 i}=-\frac{\lambda_{4}}{2 \lambda_{3}} \frac{\partial n_{i}}{\partial z}, L_{3 i}^{4}=\frac{1}{2} \frac{\partial n_{i}}{\partial z}, L_{4 i}^{4}=\frac{1}{2} \frac{\partial \ln \left|\lambda_{4}\right|}{\partial x^{i}}
\end{aligned}
$$

and

$$
\begin{aligned}
& T_{3 i}^{3}=-T_{i 3}^{3}=\frac{\partial q_{i}}{\partial z}-L_{3 i}^{3}, T_{4 i}^{3}=-T_{i 4}^{3}=-L_{4 i}^{3}, \\
& T_{3 i}^{4}=-T_{i 3}^{4}=\frac{\partial n_{i}}{\partial z}-L^{4}{ }_{3 i}, T_{4 i}^{4}=-T_{i 4}^{4}=-L_{4 i}^{4}, \\
& T_{12}^{3}=-T_{21}^{3}=\frac{\partial q_{1}}{\partial x^{2}}-\frac{\partial q_{2}}{\partial x^{1}}-q_{2} \frac{\partial q_{1}}{\partial z}+q_{1} \frac{\partial q_{2}}{\partial z}, \\
& T_{12}^{4}=-T_{21}^{4}=\frac{\partial n_{1}}{\partial x^{2}}-\frac{\partial n_{2}}{\partial x^{1}}-q_{2} \frac{\partial n_{1}}{\partial z}+q_{1} \frac{\partial n_{2}}{\partial z} .
\end{aligned}
$$

The obtained values allow us to define some na-map chains, for instance, from the Minkowski spacetime $V_{[0]}=M^{3,1}$, where it is pointed the spacetime signature $(3,1)$, to a curved one with local anisotropy, $V^{(2+2)}$, provided by a metric (88) (equvalently, a d-metric (89)).

In this Section we shall consider sets of invertible na-maps (the inversce to a natransform is also considered to be a na-map) when we could neglect quadratic terms 
like $P P$ and FF 32] in the basic na-equations (29), (31) and (33), for simplicity, taken in a nonsymetrized form:

- for $n a_{(1)}-$ maps

$$
D_{\alpha} P_{. \beta \gamma}^{\delta}-Q_{. \alpha \tau}^{\delta} P_{\beta \gamma}^{\tau}=b_{\alpha} P_{. \beta \gamma}^{\delta}+a_{\beta \gamma} \delta_{\alpha}^{\delta}
$$

- for $n a_{(2)}-$ maps the deformation $\mathrm{d}$-tensor is parametrized

$$
P_{. \alpha \beta}^{\tau}(u)=\sigma_{(\alpha} F_{\beta}^{\tau}
$$

and the basic equations are taken

$$
D_{\gamma} F_{\beta}^{\alpha}-Q_{. \tau \gamma}^{\alpha} F_{\beta}^{\tau}=\mu_{\gamma} F_{\beta}^{\alpha}+\nu_{\gamma} \delta_{\beta}^{\alpha}
$$

- for $n a_{(3)}-$ maps the deformation $\mathrm{d}$-tensor is parametrized

$$
P_{. \beta \gamma}^{\alpha}(u)=\sigma_{\beta \gamma} \varphi^{\alpha}
$$

and the basic equations

$$
D_{\beta} \varphi^{\alpha}=\nu \delta_{\beta}^{\alpha}+\mu_{\beta} \varphi^{\alpha}+\varphi^{\gamma} Q_{. \gamma \delta}^{\alpha}
$$

\subsection{Chains of $n a_{(1)}-$ maps}

We illustrate that the canonical $\mathrm{d}$-connections and $\mathrm{d}$-torsions of the mentioned vacuum metrics with local anisotropy could be induced by a chain of three $n a_{(1)}-$ transforms

$$
V_{[0]} \stackrel{n a_{1}^{[1]}}{\longrightarrow} V_{[1]} \stackrel{n a_{1}^{[2]}}{\longrightarrow} V_{[2]} \stackrel{n a_{1}^{[3]}}{\longrightarrow} V^{(2+2)} .
$$

The first step in this chain is defined by some deformations of the symmetric (22) and antisymmetric (23) parts of the $\mathrm{d}$-connection

$$
\begin{aligned}
n a_{1}^{[1]}: & { }^{[1]} \gamma_{\beta \tau}^{\alpha}={ }^{[0]} \gamma_{\beta \tau}^{\alpha}+{ }^{[1]} P_{\beta \tau}^{\alpha}, \\
{ }^{[1]} T_{\beta \tau}^{\alpha} & ={ }^{[0]} T_{\beta \tau}^{\alpha}+{ }^{[1]} Q_{\beta \tau}^{\alpha},
\end{aligned}
$$

where it is considered that on the flat background space is chosen a system of coordinates for which ${ }^{[0]} \gamma_{\beta \tau}^{\alpha}=0$ and ${ }^{[0]} T_{\beta \tau}^{\alpha}=0$. The values

$$
\left({ }^{[1]} b_{\alpha}=\partial_{\alpha} \ln \left|L^{1}{ }_{12}\right|,{ }^{[1]} a_{\beta \gamma}=0,{ }^{[1]} P_{\beta \gamma}^{\alpha}=\left\{L^{1}{ }_{12}=L^{1}{ }_{21}\right\},{ }^{[1]} Q_{\beta \gamma}^{\alpha}=0\right)
$$

solve the system of $n a_{(1)}$-equations (99). The resulting auxiliary curved space $V_{\text {[1] }}$ is provided with a d-covariant differential operator ${ }^{[1]} D_{\alpha}$, defined by the $\mathrm{d}-$-connection ${ }^{[1]} \Gamma_{\beta \tau}^{\alpha}={ }^{[1]} \gamma_{\beta \tau}^{\alpha}+{ }^{[1]} T_{\beta \tau}^{\alpha}$.

The second step in the chain (104) is parametrized by the deformations

$$
\begin{aligned}
n a_{1}^{[2]}: & { }^{[1]} \gamma_{\beta \tau}^{\alpha}={ }^{[1]} \gamma_{\beta \tau}^{\alpha}+{ }^{[2]} P_{\beta \tau}^{\alpha} \\
{ }^{[2]} T_{\beta \tau}^{\alpha} & ={ }^{[1]} T_{\beta \tau}^{\alpha}+{ }^{[2]} Q_{\beta \tau}^{\alpha}
\end{aligned}
$$


with associated values

$$
\left({ }^{[2]} b_{\alpha}=\left(L^{2}{ }_{11}\right)^{-1}{ }^{[1]} D_{\alpha} L^{2}{ }_{11},{ }^{[2]} a_{\beta \gamma}=0,{ }^{[2]} P_{\beta \gamma}^{\alpha}=\left\{L^{2}{ }_{11}\right\},{ }^{[2]} Q_{\beta \gamma}^{\alpha}=0\right)
$$

solving the system of $n a_{(1)}$-equations (99) for fixed initial data on the auxiliary space $V_{[1]}$. The resulting auxiliary curved space $V_{[2]}$ is provided with a d-covariant differential operator ${ }^{[2]} D_{\alpha}$, defined by the $\mathrm{d}$-connection ${ }^{[2]} \Gamma_{\beta \tau}^{\alpha}={ }^{[2]} \gamma_{\beta \tau}^{\alpha}{ }^{[2]} T_{\beta \tau}^{\alpha}$.

The third, final, map $n a_{1}^{[3]}$, which induces a la-spacetime $V^{(2+2)}$ with the $\mathrm{d}-$-connection (97) and d-torsions (98), could be treated as trivial $n a_{(1)}$-map with a simple deformation of the torsion structure

$$
{ }^{[3]} T_{\beta \tau}^{\alpha}={ }^{[2]} T_{\beta \tau}^{\alpha}+{ }^{[3]} Q_{\beta \tau}^{\alpha},
$$

which is given by the set of values $\left({ }^{[3]} b_{\alpha}=0,{ }^{[2]} a_{\beta \gamma}=0,{ }^{[2]} P_{\beta \gamma}^{\alpha}=0,{ }^{[2]} Q_{\beta \gamma}^{\alpha}=T_{\beta \gamma}^{\alpha}\right)$, where $T_{\beta \gamma}^{\alpha}$ has just the components (98).

So, we have proved that the vacuum Einstein fields given by a metric (88) (equivalently, by a d-metric (89), induced by a Finsler like metric, are nearly conformaly flat, being related ba chain of two $n a_{(1)}$-maps and a deformation of torsion structure with the Minkowski spacetime. On every spacetime, on the initial $V_{[0]}$, two auxiliary, $V_{[1]}$, and $V_{[2]}$, and on the final image, $V^{(2+2)}$ one holds $n a_{(1)}$-invariant conditions of type (35).

\subsection{Chains of $n a_{(2)}-$ maps}

The considered vacuum la-spacetimes could be also induced by a chain of three $n a_{(2)}$-maps from the Minkowski spacetime,

$$
V_{[0]} \stackrel{n a_{2}^{[1]}}{\longrightarrow} V_{[1]} \stackrel{n a_{2}^{[2]}}{\longrightarrow} V_{[2]} \stackrel{n a_{2}^{[3]}}{\longrightarrow} V^{(2+2)} .
$$

The first $n a_{2}-$ map from this chain is defined by some deformations of the symmetric part (22), with the deformation d-tensor parametrized as (100), and antisymmetric (23) part of the $\mathrm{d}-$-connection

$$
\begin{aligned}
n a_{2}^{[1]}: & { }^{[1]} \gamma_{\beta \tau}^{\alpha}={ }^{[0]} \gamma_{\beta \tau}^{\alpha}+{ }^{[1]} \sigma_{\beta}{ }^{[1]} F_{\tau}^{\alpha}, \\
{ }^{[1]} T_{\beta \tau}^{\alpha} & ={ }^{[0]} T_{\beta \tau}^{\alpha}+{ }^{[1]} Q_{\beta \tau}^{\alpha},
\end{aligned}
$$

where it is considered that on the flat background space is chosen a system of coordinates for which ${ }^{[0]} \gamma_{\beta \tau}^{\alpha}=0$ and ${ }^{[0]} T_{\beta \tau}^{\alpha}=0$. The values

$$
\left({ }^{[1]} F_{\tau}^{\alpha}=\left\{L_{12}^{1}\right\},{ }^{[1]} \mu_{\alpha}=\partial_{\alpha} \ln \left|L_{12}^{1}\right|,{ }^{[1]} \nu_{\beta}=0,{ }^{[1]} \sigma_{\alpha}=\delta_{\alpha}^{1},{ }^{[1]} Q_{\beta \gamma}^{\alpha}=0\right)
$$

solve the system of $n a_{(2)}$-equations (101). The resulting auxiliary curved space $V_{[1]}$ is provided with a d-covariant differential operator ${ }^{[1]} D_{\alpha}$, defined by the $\mathrm{d}-$-connection ${ }^{[1]} \Gamma_{\beta \tau}^{\alpha}={ }^{[1]} \gamma_{\beta \tau}^{\alpha}$.

The second $n a_{2}-$ map from (105) is parametrized as

$$
\begin{aligned}
n a_{2}^{[2]}: & { }^{[2]} \gamma_{\beta \tau}^{\alpha}={ }^{[1]} \gamma_{\beta \tau}^{\alpha}+{ }^{[2]} \sigma_{\beta}{ }^{[2]} F_{\tau}^{\alpha} \\
{ }^{[2]} T_{\beta \tau}^{\alpha} & ={ }^{[1]} T_{\beta \tau}^{\alpha}+{ }^{[2]} Q_{\beta \tau}^{\alpha}
\end{aligned}
$$


with the values

$$
\left({ }^{[2]} F_{\tau}^{\alpha}=\left\{L^{2}{ }_{11}\right\},{ }^{[2]} \mu_{\alpha}=\left(L^{2}{ }_{11}\right)^{-1}{ }^{[1]} D_{\alpha} L^{2}{ }_{11},{ }^{[2]} \nu_{\beta}=0,{ }^{[2]} \sigma_{\alpha}=\delta_{\alpha}^{1},{ }^{[2]} Q_{\beta \gamma}^{\alpha}=0\right)
$$

solving the system of $n a_{(2)}$-equations (101). The second resulting auxiliary curved space $V_{[2]}$ is provided with a d-covariant differential operator ${ }^{[2]} D_{\alpha}$, defined by the $\mathrm{d}-$ connection ${ }^{[2]} \Gamma_{\beta \tau}^{\alpha}={ }^{[2]} \gamma_{\beta \tau}^{\alpha}$.

The third step in the chain (105) is a trivial $n a_{2}-$ map with pure deformation of $\mathrm{d}$-torsions given by the values

$$
\left({ }^{[3]} F_{\tau}^{\alpha}=0,{ }^{[3]} \mu_{\alpha}=0,{ }^{[3]} \nu_{\beta}=0,{ }^{[3]} \sigma_{\alpha}=0,{ }^{[3]} Q_{\beta \gamma}^{\alpha}=T_{\beta \gamma}^{\alpha}\right),
$$

where the d-torsions are those from (98). We can conclude that a vacuum Einstein $V^{(2+2)}$ spacetime provided with a Finsler like induced metric of type (88) (equivalently, by a d-metric (89), could be alternatively induced by a chain of $n a_{2}-$ maps for which, on every stape, one holds the invariant conditions (36). This is a particular property of this class of $\mathrm{d}$-metrics. We shall prove in the next subsection that in a similar fashion we can consider chains of $n a_{3}$-mapa for inducing such types of vacuum la-spacetimes.

\subsection{Chains of $n a_{(3)}-$ maps}

Finaly, we elucidate the possibility of inducing vacuum Finsler like induced Einstein spaces by using chains of $n a_{(3)}-$ maps,

$$
V_{[0]} \stackrel{n a_{3}^{[1]}}{\longrightarrow} V_{[1]} \stackrel{n a_{3}^{[2]}}{\longrightarrow} V_{[2]} \stackrel{n a_{3}^{[3]}}{\longrightarrow} V^{(2+2)}
$$

Now, the first $n a_{3}-$ map is defined by some deformations of the symmetric part (22), with the deformation d-tensor parametrized as (102), and antisymmetric (23) part of the d-connection

$$
\begin{aligned}
n a_{3}^{[1]}: & { }^{[1]} \gamma_{\beta \tau}^{\alpha}={ }^{[0]} \gamma_{\beta \tau}^{\alpha}+{ }^{[1]} \sigma_{\beta \tau}{ }^{[1]} \varphi^{\alpha} \\
{ }^{[1]} T_{\beta \tau}^{\alpha} & ={ }^{[0]} T_{\beta \tau}^{\alpha}+{ }^{[1]} Q_{\beta \tau}^{\alpha},
\end{aligned}
$$

where it is considered that on the flat background space is chosen a system of coordinates for which ${ }^{[0]} \gamma_{\beta \tau}^{\alpha}=0$ and ${ }^{[0]} T_{\beta \tau}^{\alpha}=0$. The values

$$
\left({ }^{[1]} P_{\tau \beta}^{\alpha}=\left\{{ }^{[1]} \sigma_{12}{ }^{[1]} \varphi^{1}=L_{12}^{1}\right\},{ }^{[1]} \mu_{\alpha}=\partial_{\alpha}{ }^{[1]} \varphi^{1[1]} \nu=0,{ }^{[1]} \varphi^{\alpha}=\delta_{1}^{\alpha},{ }^{[1]} Q_{\beta \gamma}^{\alpha}=0\right)
$$

solve the system of $n a_{(3)}-$ equations (103). The resulting auxiliary curved space $V_{[1]}$ is provided with a d-covariant differential operator ${ }^{[1]} D_{\alpha}$, defined by the $\mathrm{d}$-connection ${ }^{[1]} \Gamma_{\beta \tau}^{\alpha}={ }^{[1]} \gamma_{\beta \tau}^{\alpha}$.

The second $n a_{3}-$ map from (106) is stated by

$$
\begin{aligned}
n a_{3}^{[2]}: & { }^{[2]} \gamma_{\beta \tau}^{\alpha}={ }^{[1]} \gamma_{\beta \tau}^{\alpha}+{ }^{[2]} \sigma_{\beta \tau}{ }^{[1]} \varphi^{\alpha} \\
{ }^{[2]} T_{\beta \tau}^{\alpha} & ={ }^{[1]} T_{\beta \tau}^{\alpha}+{ }^{[2]} Q_{\beta \tau}^{\alpha}
\end{aligned}
$$


with the values

$$
\left({ }^{[2]} P_{\tau \beta}^{\alpha}=\left\{{ }^{[2]} \sigma_{11}{ }^{[2]} \varphi^{2}=L^{2}{ }_{11}\right\},{ }^{[2]} \mu_{\alpha}={ }^{[2]} D_{\alpha}{ }^{[1]} \varphi^{2}{ }^{[2]} \nu=0,{ }^{[2]} \varphi^{\alpha}=\delta_{2}^{\alpha},{ }^{[2]} Q_{\beta \gamma}^{\alpha}=0\right)
$$

defining a solution of the system of $n a_{(3)}$-equations (103).

The third step in the chain (106) should be treated as a trivial $n a_{3}$-map when all parameters and deformations vanishes exepting a pure deformation of $\mathrm{d}$-torsions, ${ }^{[3]} Q_{\beta \gamma}^{\alpha}=T_{\beta \gamma}^{\alpha}$, where the d-torsions are those from (98).

\section{Outline of the Results}

In this paper we showed how the Einstein equations can be written with respect to anholonomic frames with associated nonlinear connection structures, when the dynamics of gravitational and matter field interactions is described by mixed sets of holonomic and anholonomic variables.

We demonstrated that by using anholonomic frames on (pseudo) Riemannian spacetimes we can model locally anisotropic interactions and structures (Finsler like and more general ones) which are defined in the framework of the general relativity theory. The very important role of definition of frame systems in Einstein gravity was emphasized by explicit examples when the tetrad coefficients are selected as to define new classes of solutions, with generic local anisotropy, of the Einstein equations. There were considered vacuum and non-vacuum gravitational fields induced by some generalized Finsler like metrics

Our principial aim in this work was to underline locally anisotropic gravitational effects and give them a rigorous geometrical spacetime description.

We elaborated the theory of nearly autoparallel locally anisotropic maps which generalizes the geometry of conformal and geodesic transforms and applied it for definition of conservation laws (via tensor integrals and/or by introducing nearly autoparallel backgrounds) on spacetimes provided with anholonomic structures. Nearly autoparallel chain resolutions of Finsler like induced vacuum Einstein fields were constructed.

\section{Acknoweledgments}

S. V. work was supported by the German Academic Exchange Service (DAAD).

\section{References}

[1] Asanov G S 1985 Finsler Geometry, Relativity and Gauge Theories (Boston: Reidel)

[2] Asanov G S and Ponomarenko S F 1988 Finsler Bundle on Space-Time. Associated Gauge Fields and Connections (Chişinău, Ştiinţa) [in Russian]

[3] Barthel W 1963 J. Angew. Math. 212120 
[4] Bejancu A 1990 Finsler Geometry and Applications (Chichester, England: Ellis Horwood)

[5] Bogoslovsky G Yu 1977 Nuov. Cim. B40 99; 116

[6] Bogoslovsky G Yu 1992 Theory of Locally Anisotropic Space-Time (Moscow, Moscow State University Publ.) [in Russian]

[7] Cartan E 1923 Sur les varietes a connexion affine et la theorie de la relativite generalises (Ann. Ex. Norm. Sup), 325 [Russion translation in the collection of works: Cartan E, 1962 Pronstranstva affinoy, proektivnoy $i$ konformnoy sveaznosti (Kazani, Kazani University Press)]

[8] Cartan E 1930 Math. Cluj 4111

[9] Cartan E 1935 La methode du repere mobile, la theories des groupes continus et les espaces generalises (Paris: Hermann)

[10] Cartan E 1935 Les Espaces de Finsler (Paris: Hermann)

[11] Cartan E 1939 Les problemes d'equivalence, in: Selectia de M. Cartan (Paris) 113

[12] Cartan E 1945 Les sytemes differentiels exterieurs et leurs applications geometriques (Paris, Actualites)

[13] Ellis G and Hawking S 1973 The Large Scale Structure of Space-Time (Cambridge: Cambridge University Press)

[14] Finsler P $1918 \ddot{U}$ ber Kurven und Flächen in Allgemeiner Rämen, Dissertation (Göttingen); reprinted 1951 (Basel: Birkhäuser)

[15] Gegenberg J and Kunstatter G 1998 Phys. Lett. B 413, 274 (1997); Phys. Rev. D 58, 124010 (1998).

[16] Goenner H F and Bogoslovsky G Yu 2000 Ann. Phys. (Leipzig) 9 Spec. Issue, 54

[17] Gottlieb I, Oproiu V and Zet G 1974 An. Şti. Univ. "Al. I. Cuza" Iasi, Sect. Ia. Mat. (N.S.) 20123

[18] Gottlieb I and Vacaru S 1996 Lagrange and Finsler Geometry, Applications to Physics and Biology, eds. P. L. Antonelli and Radu Miron (Dordrecht, Boston, London: Kluwer Academic Publishers), 209

[19] Grishchuk L P, Petrov A N and Popova A D 1984 Commun. Math. Phys. 94 379

[20] Jackiv R and Teitelboim C 1994, contributions in Quantum Theory of Gravity, ed Christense S M (Hilger, Bristol) 
[21] E. Kamke, Differential Gleichungen, Losungsmethoden und Lonsungen: I. Gewohnliche Differentialgleichungen (Lipzig, 1959).

[22] Kern J 1974 Arch. Math. 25438

[23] Lemos J P S and Sa. P 1994 Mod. Phys. Lett. A 9771

[24] Mann R B 1993 Phys. Rev. D 47, 4438

[25] Matsumoto M 1986 Foundations of Finsler Geometry and Special Finsler Spaces (Kaisisha: Shigaken)

[26] Mielke E W 1987 Geometrodynamics of Gauge Fields - On the Geometry of Yang-Mills and Gravitational Gauge Theories (Berlin: Academie Verlag)

[27] Miron R and Anastasiei M 1987 Vector Bundles. Lagrange Spaces. Application in Relativity (Academiei, Romania 1987) [in Romanian]; English translation 1996 (Bucharest: Balkan Press)

[28] Miron R and Anastasiei M 1994 The Geometry of Lagrange Spaces: Theory and Applications (Dordrecht, Boston, London: Kluwer Academic Publishers)

[29] Misner C W, Thorne K S and Wheeler J A 1973 Gravitation (Freeman)

[30] Moór A 1951 Acta Math. 8671

[31] Rund H 1959 The Differential Geometry of Finsler Spaces (Berlin: SpringerVerlag)

[32] Sinyukov N S 1979 Geodesic Maps of Riemannian Spaces (Moscow: Nauka) [in Russian]

[33] Synge J L 1960 Relativity: The General Theory (Amsterdam: North Holland Publishing Company)

[34] Vacaru S 1994 Romanian Journal of Physics 3937

[35] Vacaru S 1995 Buletinul Academiei de Ştiinţe a Republicii Moldova, Fizica şi Tehnica (Izvestya Akademii Nauk Respubliki Moldova, fizika i tehnika) 154

[36] Vacaru S 1996 J. Math. Phys 37508

[37] Vacaru S 1997 Ann. Phys. (N.Y.) 256 39; gr-qc/9604013

[38] Vacaru S 1997 Nucl. Phys. B 424 590; hep-th/9611034, 9607196, 9607195, 9607194

[39] Vacaru S 1998 J. High Energy Phys. 09(1998)011; hep-th/9807214

[40] Vacaru S 1998 Interactions, Strings, and Isotopies in Higher Order Anisotropic Superspaces (Palm Harbor: Hadronic Press); physics/9706038; dg-ga/9609004; hep-th/9810229, 9611091; gr-qc/9604017 
[41] Vacaru S 2000 gr-qc/0001020, 0001060, 0005025

[42] Vacaru S and Ostaf S 1993 Buletinul Academiei de Ştiinţe a Republicii Moldova, Fizica şi Tehnica (Izvestya Akademii Nauk Respublici Moldova, fizika i tehninka) 34

[43] Vacaru S and Ostaf S 1996 Lagrange and Finsler Geometry, eds. P. L. Antonelli and R. Miron, (Dordrecht, Boston, London: Kluwer Academic Publishers), 241

[44] Vacaru S, Ostaf S and Goncharenko Yu 1994 Romanian J. Physics 39199

[45] Will C M 1993 Theory and Experiment in Gravity Physics, revised version (Cambridge University Press)

[46] DeWitt B S 1965 Dynamical Theory of Groups and Fields (New York: Gordon and Breach) 